\title{
LOGARITHMIC ABELIAN VARIETIES, PART IV: PROPER MODELS
}

\author{
TAKESHI KAJIWARA, \\ KAZUYA KATO, AND CHIKARA NAKAYAMA
}

\section{To Professor Luc Illusie}

\begin{abstract}
This is part IV of our series of articles on log abelian varieties. In this part, we study the algebraic theory of proper models of log abelian varieties.
\end{abstract}

\section{Contents}

0. Introduction . . . . . . . . . . . . . . . . . . . . . . . 10

1. Weak $\log$ abelian varieties . . . . . . . . . . . . . . . . . . . . 11

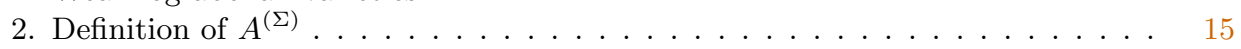

3. Complete fans . . . . . . . . . . . . . . . . . . . . . . 18

4. Models in the case of constant degeneration . . . . . . . . . . . . . . . 18

5. A weak $\log$ abelian variety is a két sheaf . . . . . . . . . . . . . . . . . . 20

6. Algebraic spaces with fs $\log$ structure . . . . . . . . . . . . . . . . 23

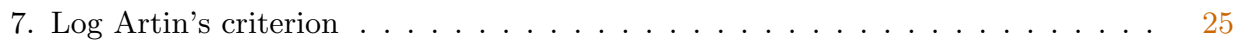

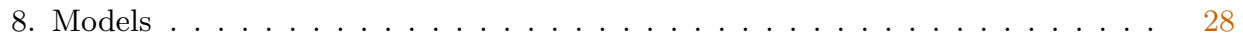

9. Weak $\log$ abelian varieties over limits . . . . . . . . . . . . . . . . . . . 32

10. Weak $\log$ abelian varieties are $\log$ algebraic spaces in some sense . . . . . . . 38

11. Valuative criterion for weak $\log$ abelian varieties . . . . . . . . . . . . . . 39

12. Proper models with constant degeneration . . . . . . . . . . . . . . . . . . . 42

13. Weak $\log$ abelian varieties over complete discrete valuation rings, I . . . . . . . 45

14. Weak log abelian varieties over complete discrete valuation rings, II . . . . . . 47

15. Weak $\log$ abelian varieties over complete discrete valuation rings, III . . . . . . 48

16. Weak log abelian varieties over complete discrete valuation rings, IV . . . . . . 51

17. Proper models . . . . . . . . . . . . . . . . . . . . 56

18. Torsion points of weak $\log$ abelian varieties . . . . . . . . . . . . . . . 57

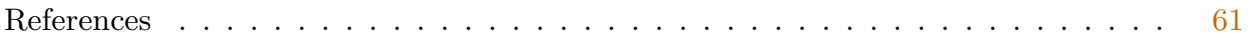

Received September 1, 2013. Revised May 14, 2014. Accepted May 19, 2014.

2010 Mathematics Subject Classification. Primary 14K10; Secondary 14J10, 14D06. 


\section{$\S 0$. Introduction}

This is part IV of our series of articles on log abelian varieties.

Degenerating abelian varieties cannot preserve group structures, properness, and smoothness at the same time. However, in a log world, degenerating abelian varieties can become group objects called log abelian varieties, which behave like proper smooth objects. For the idea of our theory, see the introductory section of part II [9, Section 1].

In part I [8], we studied a complex analytic theory of log abelian varieties. In part II [9], we started to study an algebraic theory of log abelian varieties. In part III [10], we illustrated our theory in the case of log elliptic curves. In this part IV, we continue to study an algebraic theory of log abelian varieties. The main subject of this part is to construct proper models of log abelian varieties.

We plan to study polarizations and projective models in part V (forthcoming). In the remaining parts of this series, one of the main subjects should be moduli spaces of log abelian varieties.

In this part, we work with the notion of weak log abelian varieties, which is a slight generalization of that of log abelian varieties, and which is more natural for the study of proper models. See Section 1 for the definition of weak log abelian varieties.

Our first main result is, in rough form, the following.

Theorem 0.1. For any weak log abelian variety $A$ over an fs log scheme and for any fan $\Sigma$, the $\Sigma$-part $A^{(\Sigma)}$ of $A$ is an algebraic space.

This is proved in Section 8 by using a log version of the Artin criterion on representability of a functor by an algebraic space (see Section 7).

The second main theorem is, roughly speaking, the existence of proper models of weak log abelian varieties, as follows.

THEOREM 0.2. For any weak log abelian variety $A$ over an $f s$ log scheme and for any complete fan $\Sigma$, the $\Sigma$-part $A^{(\Sigma)}$ of $A$ is proper.

By the existence of a complete fan (see [8, 5.2.1]), this theorem shows that we have always a proper model of $A$ étale locally on the base. Moreover, a proper model exists globally under some situations (see Sections 2.4 and 2.5).

Theorem 0.2 is in Section 17. To prove this, we use the valuative criterion. So we need the detailed analysis of the category of weak log abelian varieties 
over a trait (see Sections 13-16). This story continues in a forthcoming part of this series of articles.

Terminology. A morphism $f: X \rightarrow Y$ of fs log schemes is strict if the induced homomorphism of $\log$ structures $f^{*} M_{Y} \rightarrow M_{X}$ is an isomorphism, and $f$ is affine (resp., finite, resp., proper) if its underlying morphism of schemes is affine (resp., finite, resp., proper).

\section{$\S 1$. Weak log abelian varieties}

As we said in the Introduction, our main subject is the study of proper models of log abelian varieties. To this end, it is natural and convenient to generalize the notion log abelian variety. We introduce and work with the notion weak log abelian varieties instead of log abelian varieties. This notion weak log abelian variety is an algebraic analogue of the notion log complex torus (see [8]) all of whose fibers are algebraic (see Section 1.8).

1.1. To define a weak log abelian variety, we first review the notion admissible pairing (see [8], [9]).

Let $X$ and $Y$ be finitely generated free $\mathbb{Z}$-modules, let $\mathcal{S}$ be an fs monoid, and let

$$
\langle,\rangle: X \times Y \rightarrow \mathcal{S}^{\mathrm{gp}}
$$

be a $\mathbb{Z}$-bilinear form. For a face $\sigma$ of $\mathcal{S}$, let $X_{\sigma}$ be the subgroup of $X$ consisting of all elements $x$ of $X$ such that $\langle x, Y\rangle$ is contained in $\sigma^{\mathrm{gp}}$. The subgroup $Y_{\sigma}$ of $Y$ is defined similarly.

We say that $\langle$,$\rangle is admissible (precisely, \mathcal{S}$-admissible) if the following condition is satisfied: for any face $\sigma$ of $\mathcal{S}$ and any homomorphism $N: \mathcal{S} \rightarrow$ $\mathbb{R}_{\geq 0}$ into the additive monoid $\mathbb{R}_{\geq 0}$, if we write the face $\sigma \cap N^{-1}(0)$ of $\mathcal{S}$ as $\tau$, then the induced pairing of $\mathbb{R}$-linear spaces by $N$

$$
\left(X_{\sigma} / X_{\tau}\right)_{\mathbb{R}} \times\left(Y_{\sigma} / Y_{\tau}\right)_{\mathbb{R}} \rightarrow \mathbb{R}
$$

is nondegenerate.

Equivalently, it is admissible if the following condition is satisfied: for any face $\sigma$ of $\mathcal{S}$ and any homomorphism $N: \sigma \rightarrow \mathbb{R}_{\geq 0}$, the induced pairing of $\mathbb{R}$-linear spaces by $N$

$$
\left(X_{\sigma} / X_{\tau}\right)_{\mathbb{R}} \times\left(Y_{\sigma} / Y_{\tau}\right)_{\mathbb{R}} \rightarrow \mathbb{R}
$$

is nondegenerate, where $\tau$ is the face $N^{-1}(0)$ of $\sigma$. 
1.2. Let $S$ be an fs $\log$ scheme. We denote by

$$
(\mathrm{fs} / S)
$$

the category of fs log schemes over $S$. We endow it with the strict étale topology and denote it by (fs/S)ét (see [9, 1.2]).

We denote by $\mathbb{G}_{m, \log }$ or by $\mathbb{G}_{m, \log , S}$ (resp., by $\mathbb{G}_{m}$ or by $\mathbb{G}_{m, S}$ ) the sheaf of abelian groups on $(\mathrm{fs} / S)_{\text {ét }}$ defined by $\mathbb{G}_{m, \log }(T)=\Gamma\left(T, M_{T}^{\mathrm{gp}}\right)$ (resp., $\left.\mathbb{G}_{m}(T)=\Gamma\left(T, \mathcal{O}_{T}^{\times}\right)\right)$.

Let $X$ and $Y$ be finitely generated free $\mathbb{Z}$-modules, and let

$$
\langle,\rangle: X \times Y \rightarrow\left(\mathbb{G}_{m, \log } / \mathbb{G}_{m}\right)_{S}
$$

be a $\mathbb{Z}$-bilinear form. We say that $\langle$,$\rangle is admissible if, for any s \in S$, the induced pairing $X \times Y \rightarrow\left(M_{S}^{\mathrm{gp}} / \mathcal{O}_{S}^{\times}\right)_{\bar{s}}$ is $\left(M_{S} / \mathcal{O}_{S}^{\times}\right)_{\bar{s}}$-admissible.

1.3. Let $S$ be an fs $\log$ scheme. Let $\langle\rangle:, X \times Y \rightarrow\left(\mathbb{G}_{m, \log } / \mathbb{G}_{m}\right)_{S}$ be an admissible pairing. Here and hereafter, we regard the sheaves as those on $(\mathrm{fs} / S)_{\text {ét }}$. Consider the sheaf of abelian groups $\mathcal{H o m}\left(X, \mathbb{G}_{m, \log } / \mathbb{G}_{m}\right)$ on $S$. The admissible pairing gives a homomorphism $Y \rightarrow \mathcal{H o m}\left(X, \mathbb{G}_{m, \log } / \mathbb{G}_{m}\right)$. We define a subsheaf

$$
\mathcal{Q}:=\mathcal{H} o m\left(X, \mathbb{G}_{m, \log } / \mathbb{G}_{m}\right)^{(Y)}
$$

of groups, which plays an important role in our theory. Let $U \in($ fs $/ S)$. A section $\varphi$ of $\mathcal{H o m}\left(X, \mathbb{G}_{m}, \log / \mathbb{G}_{m}\right)$ over $U$ belongs to this subsheaf $\mathcal{Q}$ of groups if and only if, for any $u \in U$ and for any $x \in X_{\bar{u}}$, there exist $y, y^{\prime} \in Y_{\bar{u}}$ such that $\langle x, y\rangle\left|\varphi_{\bar{u}}(x)\right|\left\langle x, y^{\prime}\right\rangle$, that is, such that $\varphi_{\bar{u}}(x) /\langle x, y\rangle$ and $\left\langle x, y^{\prime}\right\rangle / \varphi_{\bar{u}}(x)$ belong to $\left(M_{U} / \mathcal{O}_{U}^{\times}\right)_{\bar{u}}$ in $\left(M_{U}^{\mathrm{gp}} / \mathcal{O}_{U}^{\times}\right)_{\bar{u}}$.

We denote by $\bar{Y}$ the image of $Y$ in $\mathcal{H o m}\left(X, \mathbb{G}_{m, \log } / \mathbb{G}_{m}\right)$, which is contained in $\mathcal{Q}$.

1.4. We briefly review the notion $\log 1$-motif and some related definitions. See [9, Sections 2.1, 2.3, and 3.2] for the details.

Let $S$ be an fs log scheme. Let $G$ be a commutative group scheme over the underlying scheme of $S$ which is an extension of an abelian scheme $B$ over $S$ by a torus $T$ over $S$. We regard $G, B$, and $T$ as sheaves of abelian groups on $(\mathrm{fs} / S)_{\text {ét }}$. Let $X:=\mathcal{H o m}\left(T, \mathbb{G}_{m}\right)$, and let $T_{\log }=\mathcal{H o m}\left(X, \mathbb{G}_{m, \log }\right)$. Let $G_{\log }$ be the pushout of $G \leftarrow T \hookrightarrow T_{\log }$ in the category of sheaves of abelian groups on (fs/ $S$ ) ét. 
A $\log 1$-motif $\left[Y \rightarrow G_{\log }\right]$ over $S$ is a complex of sheaves of abelian groups on (fs $/ S$ )ét with $G_{\log }$ degree 0 , where $Y$ is a locally constant sheaf of finitely generated and free abelian groups, and $G$ is a commutative group scheme over the underlying scheme of $S$ which is an extension of an abelian scheme by a torus.

Assume that we are given a log 1-motif as above. Assume that the canonical pairing

$$
X \times Y \rightarrow X \times\left(G_{\log } / G\right) \cong X \times\left(T_{\log } / T\right) \rightarrow \mathbb{G}_{m, \log } / \mathbb{G}_{m}
$$

is admissible, where $X=\mathcal{H} o m\left(T, \mathbb{G}_{m}\right)$.

Let

$$
G_{\log }^{(Y)} \subset G_{\log }
$$

be the inverse image of $\mathcal{Q}$ (see Section 1.3) under $G_{\log } \rightarrow G_{\log } / G \cong \mathcal{H o m}(X$, $\left.\mathbb{G}_{m, \log } / \mathbb{G}_{m}\right)$.

1.5. Let $(P)$ be a property of morphisms of fs log schemes. We say that a morphism $F_{1} \rightarrow F_{2}$ of contravariant functors (fs $\left./ S\right) \rightarrow$ (Set) is represented by morphisms with the property $(P)$ if, for any fs log scheme $V$ over $S$ and any morphism $V \rightarrow F_{2}$ over $S$, the fiber product $F_{1} \times{ }_{F_{2}} V$ is represented by an fs $\log$ scheme over $S$ and the morphism $F_{1} \times_{F_{2}} V \rightarrow V$ obtained by base change has the property $(P)$.

Below, we consider the case where the morphism is the diagonal one $\Delta: F_{1} \rightarrow F_{1} \times F_{1}$. In this case, if $(P)$ is stable under base changes in the category of fs log schemes, it is formally shown that $\Delta$ is represented by morphisms with the property $(P)$ if and only if the following holds: for any fs $\log$ schemes $U$ and $V$ over $S$ and for any morphisms $U \rightarrow F_{1}$ and $V \rightarrow F_{1}$, the fiber product $U \times_{F_{1}} V$ is represented by an fs $\log$ scheme over $S$ and the natural morphism $U \times_{F_{1}} V \rightarrow U \times_{S} V$ has the property $(P)$.

Definition 1.6. A weak log abelian variety over an fs log scheme $S$ is a sheaf of abelian groups $A$ on $(\mathrm{fs} / S)_{\text {ét }}$ satisfying the following three conditions.

(1) For any $s \in S$, the pullback of $A$ to $(\mathrm{fs} / \bar{s})_{\text {ét }}$ is isomorphic to $G_{\log }^{(Y)} / Y$ for an admissible and nondegenerate $\log 1$-motif $\left[Y \rightarrow G_{\log }\right]$ (see Section 1.4).

(2) Étale locally on $S$, there are a semiabelian scheme $G$ over $S$, finitely generated free $\mathbb{Z}$-modules $X$ and $Y$, an admissible pairing $\langle\rangle:, X \times Y \rightarrow$ 
$\left(\mathbb{G}_{m, \log } / \mathbb{G}_{m}\right)_{S}$ on $(\mathrm{fs} / S)$, and an exact sequence

$$
0 \rightarrow G \rightarrow A \rightarrow \mathcal{Q} / \bar{Y} \rightarrow 0
$$

where $\bar{Y}$ denotes the image of $Y$ in $\mathcal{Q}=\mathcal{H o m}\left(X, \mathbb{G}_{m, \log } / \mathbb{G}_{m}\right)^{(Y)}$ as in Section 1.3.

(3) The diagonal morphism $A \rightarrow A \times A$ is represented by finite morphisms.

If we replace "admissible and nondegenerate" in condition (1) by "polarizable" (see [9, Definition 2.8]), we get the definition of $\log$ abelian variety (see [9, Section 4]).

Since the polarizability implies the admissibility and the nondegeneracy (see [9, Example 7.2(1)]), a log abelian variety is a weak log abelian variety.

1.7. We can develop the theory of weak log abelian varieties in a parallel way to that of log abelian varieties in [9].

Let $S$ be an fs log scheme. The semiabelian part $G$ and the canonical pairing $\bar{X} \times \bar{Y} \rightarrow \mathbb{G}_{m, \log } / \mathbb{G}_{m}$ for a weak log abelian variety $A$ are globally defined in the same way as in [9, Sections 4.4, 9.2, and 9.3]. A weak log abelian variety with constant degeneration over $S$ is a sheaf of abelian groups on

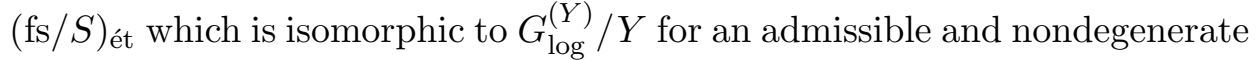
$\log 1$-motif $\left[Y \rightarrow G_{\log }\right]$.

By $[9,8.1]$, the category of weak log abelian varieties with constant degeneration is equivalent to that of log 1-motifs whose pairing is admissible and nondegenerate. A weak log abelian variety with constant degeneration is a weak log abelian variety. A weak log abelian variety is with constant degeneration if and only if the rank of the torus part of a fiber of the semiabelian part is locally constant on $S$. If the sheaf $M_{S} / \mathcal{O}_{S}^{\times}$on $S_{\text {ét }}$ is locally constant, any weak $\log$ abelian variety over $S$ is with constant degeneration. These results are analogs of [9, Proposition 4.5 and Theorem 4.6] and are proved similarly.

1.8. We can develop the analytic theory over $\mathbb{C}$ of weak log abelian varieties. Recall that, for an fs $\log$ analytic space $S$ over $\mathbb{C}$, a log abelian variety over $S$ in the sense of [8] is a log complex torus $A$ over $S$ in the sense of [8] such that for each $s \in S$, the pullback of $A$ to $s$ comes from a log abelian variety on the fs $\log$ scheme $s$. (Note that $s=\operatorname{Spec}(\mathbb{C})$ with an fs log structure, and hence $s$ can be regarded as an fs log scheme over $\mathbb{C}$.) Here the fact that $F$ comes from $F^{\prime}$ means that the sheaf $F$ on the site of fs log analytic spaces over $s$ is the inverse image of the sheaf $F^{\prime}$ on the site of fs 
$\log$ schemes over $s$ with the strict étale topology (see [9, Section 4.9]). We define a weak log algebraic variety over $S$ as a log complex torus $A$ over $S$ such that, for each $s \in S$, the pullback of $A$ to $s$ comes from a weak log abelian variety over the fs log scheme $s$.

Let $H$ be the log Hodge structure of weight -1 over an fs log analytic space $S$ corresponding to a $\log$ complex torus $A$ over $S$. Then, $A$ is a $\log$ abelian variety (resp., weak log abelian variety) over $S$ if and only if, for each $s \in S$, the pullback $\left.H\right|_{s}$ of $H$ to $s$ has the following property: $\left.H\right|_{s}$ is polarizable (resp., the graded quotient $\operatorname{gr}_{-1}^{W(s)}\left(\left.H\right|_{s}\right.$ ) with respect to the weight filtration $W(s)$ at $s$ is polarizable).

We can say that, for an fs log analytic space $S$, a log abelian variety (resp., a weak log abelian variety) over $S$ is a log complex torus over $S$ all of whose fibers are polarizable (resp., algebraic). In fact, if $A$ is a weak $\log$ abelian variety over $S$, by definition the pullback of $A$ to each $s \in S$ comes from an algebraic object, that is, a weak log abelian variety over the fs log scheme $s$. Conversely, a $\log$ complex torus $A$ over $S$ is a weak log abelian variety if, for each $s \in S$, the complex torus corresponding to $\operatorname{gr}_{-1}^{W(s)}\left(\left.H\right|_{s}\right)$ is algebraic (i.e., it is an abelian variety).

\section{§2. Definition of $A^{(\Sigma)}$}

By definition, a weak log abelian variety $A$ over an fs log scheme $S$ is an étale sheaf on the big site of $S$. In this section, we define a certain type of subsheaf $A^{(\Sigma)}$ of $A$, which is proved to be representable under some mild conditions (see Sections 4 and 8). We call these models of $A$, and they are the main subject of this article and the next installment of this series of papers.

2.1. Let $S$ be an fs $\log$ scheme. Let $\langle\rangle:, X \times Y \rightarrow\left(\mathbb{G}_{m, \log } / \mathbb{G}_{m}\right)_{S}$ be an admissible pairing (see Section 1.2).

Then, étale locally on $S$, there exist a homomorphism from an fs monoid

$$
\mathcal{S} \rightarrow M_{S} / \mathcal{O}_{S}^{\times}
$$

and an admissible pairing

$$
X \times Y \rightarrow \mathcal{S}^{\mathrm{gp}}
$$

which induce the above admissible pairing (see [9, Section 7.7]).

In the following, we assume that these data exist and fix them. 
2.2. Let $C$ be the subcone of $\operatorname{Hom}(\mathcal{S}, \mathbb{N}) \times \operatorname{Hom}(X, \mathbb{Z})$ defined as

$$
C=\left\{(N, l) \mid l\left(X_{\operatorname{ker}(N)}\right)=0\right\}
$$

(see [8, Section 3.4.2]). By a finitely generated subcone of $C$, we mean a finitely generated submonoid $\Delta$ of the additive monoid $C$ such that for any $a \in C$ satisfying $n a \in \Delta$ for some $n \geq 1$, we have $a \in \Delta$.

For a positive rational number $a$, we denote by $a \Delta$ the subcone of $C$ spanned by the intersection of $C$ and the image of $\Delta$ by the map $C \rightarrow$ $C_{\mathbb{Q} \geq 0} ;(N, l) \mapsto(N, a l)$, where $C_{\mathbb{Q}_{\geq 0}}$ is the $\mathbb{Q} \geq 0$-monoid spanned by $C$ in $\operatorname{Hom}(\mathcal{S}, \mathbb{Q} \geq 0) \times \operatorname{Hom}(X, \mathbb{Q})$.

2.3. For a finitely generated subcone $\Delta$ of $C$, define the subsheaf

$$
\bar{V}(\Delta) \subset \mathcal{H o m}\left(X, \mathbb{G}_{m, \log } / \mathbb{G}_{m}\right)^{(Y)}
$$

as follows. For an fs log scheme $U$ over $S$,

$$
\begin{aligned}
\bar{V}(\Delta)(U)= & \left\{\varphi \in \mathcal{H} \text { om }\left(X, \mathbb{G}_{m, \log } / \mathbb{G}_{m}\right)(U) \mid\right. \\
& \left.\mu \varphi(x) \in M_{U} / \mathcal{O}_{U}^{\times} \text {for any }(\mu, x) \in \Delta^{\vee}\right\},
\end{aligned}
$$

where $\Delta^{\vee}$ is the dual cone of $\Delta$ in $\mathcal{S}^{\mathrm{gp}} \times X$; that is,

$$
\Delta^{\vee}=\left\{(\mu, x) \in \mathcal{S}^{\mathrm{gp}} \times X \mid N^{\mathrm{gp}}(\mu)+l(x) \geq 0 \text { for all }(N, l) \in \Delta\right\} .
$$

Recall that we have

$$
\mathcal{H o m}\left(X, \mathbb{G}_{m, \log } / \mathbb{G}_{m}\right)^{(Y)}=\bigcup_{\Delta} \bar{V}(\Delta),
$$

where $\Delta$ ranges over all finitely generated subcones of $C$ (see [9, Section 7.7]).

Let $\sigma$ be a finitely generated $\mathbb{Q}_{\geq 0}$-submonoid of $C_{\mathbb{Q} \geq 0}$. Then, by abuse of notation, we denote $\bar{V}(\sigma \cap C)$ by $\bar{V}(\sigma)$.

Further, for simplicity, we sometimes denote $\bar{V}(\sigma)$ just by $[\sigma]$.

2.4. Let $S$ be an fs $\log$ scheme, and let $A$ be a weak log abelian variety over $S$. Then, by Definition 1.6, étale locally on $S$, there are a semiabelian scheme $G$ over $S$, an admissible pairing $\langle\rangle:, X \times Y \rightarrow\left(\mathbb{G}_{m, \log } / \mathbb{G}_{m}\right)_{S}$ on (fs $/ S$ ), and an exact sequence

$$
0 \rightarrow G \rightarrow A \rightarrow \mathcal{Q} / \bar{Y} \rightarrow 0
$$


We assume that they globally exist.

Let $\widetilde{A}$ be the fiber product of

$$
A \rightarrow \mathcal{Q} / \bar{Y} \leftarrow \mathcal{Q}
$$

Then, we have an exact sequence

$$
0 \rightarrow G \rightarrow \widetilde{A} \rightarrow \mathcal{Q} \rightarrow 0
$$

2.5. Further, we assume that the data $\mathcal{S} \rightarrow M_{S} / \mathcal{O}_{S}^{\times}$and $X \times Y \rightarrow \mathcal{S}^{\text {gp }}$ for $\langle\rangle:, X \times Y \rightarrow\left(\mathbb{G}_{m, \log } / \mathbb{G}_{m}\right)_{S}$ as in Section 2.1 also exist.

Let $C$ be the cone as in Section 2.2.

Let $\Delta$ be a finitely generated subcone of $C$.

We define the subsheaf $\widetilde{A}^{(\Delta)}$ of $\widetilde{A}$ as the pullback of $\bar{V}(\Delta)$ by $\widetilde{A} \rightarrow \mathcal{Q}$.

We define the subsheaf $A^{(\Delta)}$ of $A$ as the pullback of the image of $\bar{V}(\Delta)$ in $\mathcal{Q} / \bar{Y}$ by $A \rightarrow \mathcal{Q} / \bar{Y}$.

2.6. Let the situation be as in Sections 2.4 and 2.5. In particular, $C \subset$ $\operatorname{Hom}(\mathcal{S}, \mathbb{N}) \times \operatorname{Hom}(X, \mathbb{Z})$ is the cone as in Section 2.2.

A fan $\Sigma$ in $C$ is by definition a fan in $\operatorname{Hom}\left(\mathcal{S}^{\mathrm{gp}} \times X, \mathbb{Q}\right)$ whose support is contained in $C_{\mathbb{Q} \geq 0}$.

We define the subsheaf of $\mathcal{Q}=\mathcal{H} o m\left(X, \mathbb{G}_{m, \log } / \mathbb{G}_{m}\right)^{(Y)}$ (see Section 1.3) associated to $\Sigma$ as

$$
\mathcal{Q}^{(\Sigma)}:=\mathcal{H} \operatorname{om}\left(X, \mathbb{G}_{m, \log } / \mathbb{G}_{m}\right)^{(\Sigma)}:=\bigcup_{\Delta \in \Sigma} \bar{V}(\Delta) .
$$

We define the subsheaf $\widetilde{A}^{(\Sigma)}$ of $\widetilde{A}$ as the pullback of $\mathcal{Q}^{(\Sigma)}$ by $\widetilde{A} \rightarrow \mathcal{Q}$.

Assume that $\Sigma$ is stable under the action of $Y$ on $C$, where $y \in Y$ acts on $C$ by $(N, l) \mapsto(N, l+N(\langle-, y\rangle))$.

Then we define the subsheaf $A^{(\Sigma)}$ of $A$ as the pullback of the image of $\mathcal{Q}^{(\Sigma)}$ in $\mathcal{Q} / \bar{Y}$ by $A \rightarrow \mathcal{Q} / \bar{Y}$.

We call these subsheaves $\Sigma$-models or $\Sigma$-parts of $\widetilde{A}$ and $A$, respectively.

REMARK 2.7. As was stated in Section 1.7, for a weak log abelian variety $A$ over $S$, the semiabelian scheme $G$, the sheaves of abelian groups $\bar{X}$ and $\bar{Y}$, and the pairing $\bar{X} \times \bar{Y} \rightarrow \mathbb{G}_{m, \log } / \mathbb{G}_{m}$ exist globally on $S$ canonically. By this and by the construction in Section $2.4 \widetilde{A}$ also exists globally on $S$ canonically. We have $\widetilde{A}=G_{\log }^{(Y)}$ in the case of constant degeneration. 


\section{§3. Complete fans}

3.1. First we review the definition of a complete fan in [8, Section 5.3.1].

Let the notation be as in Sections 2.1 and 2.2. A fan $\Sigma$ in $C$ is complete if the following three conditions are satisfied:

(1) the support of $\Sigma$ coincides with $C_{\mathbb{Q}_{\geq 0}}$,

(2) $\Sigma$ is stable under the action of $Y$,

(3) $\Sigma$ has only finitely many $Y$-orbits.

Here $y \in Y$ acts on $C$ by $(N, l) \mapsto(N, l+N(\langle-, y\rangle))$.

A complete fan exists (see [8, Theorem 5.2.1]).

Proposition 3.2. Let the situation be as in Sections 2.4 and 2.5. Assume that $\Gamma\left(S, M_{S} / \mathcal{O}_{S}^{\times}\right)$is finitely generated. If $\Sigma$ is complete, for any $\varphi \in A(S)$ there is a log modification $S^{\prime}$ of $S$ such that $\left.\varphi\right|_{S^{\prime}}$ belongs to $A^{(\Sigma)}\left(S^{\prime}\right)$.

Here a morphism $S^{\prime} \rightarrow S$ is a log modification if, strict étale locally on $S$, it is the base change of a birational proper equivariant morphism of toric varieties (see [11, Section 9], [8, Lemma 4.6.6], [14, Section 3.6]).

Proof of Proposition 3.2. It is enough to show that, for any finitely generated subcone $\Delta$ of $C$ and for any $\varphi \in \bar{V}(\Delta)(S)$ (see Section 2.3), there exist a finite number of cones $\sigma_{i}$ of $\Sigma$ and a $\log$ modification $S^{\prime}$ of $S$ such that $\left.\varphi\right|_{S^{\prime}} \in \bigcup_{i} \bar{V}\left(\sigma_{i}\right)\left(S^{\prime}\right)$.

We take any finite number of $\sigma_{i}$ such that $\Delta \subset \bigcup_{i} \sigma_{i}$ and prove that there is a $\log$ modification as above.

Let us denote by $P$ the fs monoid $\Gamma\left(S, M_{S} / \mathcal{O}_{S}^{\times}\right)$. Using the definition of $\bar{V}(\Delta)$, we have the homomorphism $\Delta^{\vee} \rightarrow P ;(\mu, x) \mapsto \mu \varphi(x)$. Taking its dual, we get $P^{\vee} \rightarrow \Delta \subset \bigcup_{i} \sigma_{i}$. Let $R_{i}$ be the dual monoid of the inverse image of $\sigma_{i}$ to $P^{\vee}$ for each $i$. Taking the duals again, we get a homomorphism $\left(\sigma_{i} \cap C\right)^{\vee} \rightarrow R_{i} ;(\mu, x) \mapsto \mu \varphi(x) \in R_{i}$, so the section $\varphi_{i}=\left.\varphi\right|_{S_{i}}$ belongs to $\bar{V}\left(\sigma_{i}\right)\left(S_{i}\right) \subset \bigcup_{i} \bar{V}\left(\sigma_{i}\right)\left(S_{i}\right)$, where $S_{i}$ is the partial log modification of $S$ defined by $R_{i} \supset P$. Gluing $S_{i}$ and $\varphi_{i}$, we have a $\log$ modification $S^{\prime}=\bigcup_{i} S_{i}$ of $S$ such that $\left.\varphi\right|_{S^{\prime}}$ is in $\bigcup_{i} \bar{V}\left(\sigma_{i}\right)\left(S^{\prime}\right)$.

\section{$\S 4$. Models in the case of constant degeneration}

In this section and Section 8, we prove the representability of the models of weak $\log$ abelian varieties. In this section, we treat the case of constant degeneration. In Section 8, we prove the general case by using this case and the log Artin's criterion in Section 7. 
TheOREM 4.1. Let $S$ be an fs log scheme, and let $A$ be a weak log abelian variety over $S$. Assume that there are an exact sequence

$$
0 \rightarrow G \rightarrow A \rightarrow \mathcal{H} \operatorname{om}\left(X, \mathbb{G}_{m, \log } / \mathbb{G}_{m}\right)^{(Y)} / \bar{Y} \rightarrow 0
$$

a homomorphism $\mathcal{S} \rightarrow M_{S} / \mathcal{O}_{S}^{\times}$, and an admissible pairing $X \times Y \rightarrow \mathcal{S}^{\mathrm{gp}}$ as in Sections 2.4 and 2.5. Let $C \subset \operatorname{Hom}(\mathcal{S}, \mathbb{N}) \times \operatorname{Hom}(X, \mathbb{Z})$ be as in Section 2.2, and let $\Sigma$ be a fan in $C$.

Assume that $M_{S} / \mathcal{O}_{S}^{\times}$is locally constant. Then we have the following.

(1) The $\Sigma$-part $\widetilde{A}^{(\Sigma)}$ of $\widetilde{A}$ (see Section 2.6) is represented by a log smooth fs log scheme over $S$.

(2) Assume that $\Sigma$ is stable under the action of $Y$ (see Section 2.6). Assume further the following condition:

(*) For any $y \in Y$ and $\sigma \in \Sigma$, the action of $y$ on $(y \cdot \sigma) \cap \sigma$ is trivial. Then $A^{(\Sigma)}$ is represented by a log smooth $f$ s log scheme over $S$.

Corollary 4.2. There is a model $A^{(\Sigma)}$ of $A$ which is a proper scheme over $S$.

REMARK 4.3. In the next part of this series of articles, we prove that if $A$ is polarizable, then there is a model which is a projective scheme.

4.4. For the proofs of Theorem 4.1 and Corollary 4.2, we introduce some notation. Let the situation be as in Section 2.1. For a finitely generated subcone $\Delta$ of $C$, define the subsheaf

$$
V(\Delta) \subset \mathcal{H o m}\left(X, \mathbb{G}_{m, \log }\right)
$$

as the pullback of $\bar{V}(\Delta)$ by the projection $\mathcal{H o m}\left(X, \mathbb{G}_{m, \log }\right) \rightarrow \mathcal{H o m}(X$, $\left.\mathbb{G}_{m, \log } / \mathbb{G}_{m}\right)$.

Let

$$
\mathcal{H o m}\left(X, \mathbb{G}_{m, \log }\right)^{(\Sigma)}=\bigcup_{\Delta \in \Sigma} V(\Delta) .
$$

If $M_{S} / \mathcal{O}_{S}^{\times}$is locally constant, then $\mathcal{H o m}\left(X, \mathbb{G}_{m, \log }\right)^{(\Sigma)}$ is represented by an fs log scheme (see [9, Section 1.5]). We denote it by

$$
T_{\log }^{(\Sigma)}
$$

for short. Note that, in the totally degenerate case, that is, the case where $G$ is a torus, it is nothing but $\widetilde{A}^{(\Sigma)}$. 
4.5. We prove Theorem 4.1 and Corollary 4.2 .

We prove Theorem 4.1(1). Over $S$, we have an exact sequence

$$
0 \rightarrow T \rightarrow G \rightarrow B \rightarrow 0
$$

where $T$ is a torus and $B$ is an abelian variety. Pushing out this sequence by the natural map $T \rightarrow T_{\log }^{(\Sigma)}$, we have another exact sequence

$$
0 \rightarrow T_{\log }^{(\Sigma)} \rightarrow \widetilde{A}^{(\Sigma)} \rightarrow B \rightarrow 0
$$

that is, $\widetilde{A}^{(\Sigma)}$ is a toroidal enlargement of the $T$-torsor $G$ over $B$. Hence, it is represented by a log smooth fs log scheme.

We prove Theorem 4.1(2) by reducing it to Theorem 4.1(1). First note that $A^{(\Sigma)}=\widetilde{A}^{(\Sigma)} / Y$. If the condition $(*)$ is satisfied, the intersection $V(y$. $\sigma) \cap V(\sigma)$ is empty for any $y$ in $Y \backslash\{0\}$ and any $\sigma \in \Sigma$ so that $A^{(\Sigma)}$ is locally isomorphic to $\widetilde{A}^{(\Sigma)}$, and Theorem 4.1(2) is reduced to Theorem 4.1(1).

Corollary 4.2 is shown by the fact that there always exists a complete fan satisfying $(*)$ in Theorem 4.1(2). This is proved in [8, Theorem 5.2.1]. (Note that for any complete fan $\Sigma$, for a sufficiently large $n>0$, the fan $\frac{1}{n} \Sigma:=$ $\left\{\frac{1}{n} \sigma \mid \sigma \in \Sigma\right\}$ satisfies $(*)$, which is also complete. This gives an alternative proof when we are given a fan which is only complete.)

REMARK 4.6. The authors do not know if the condition $(*)$ in Theorem $4.1(2)$ is indispensable or not. But, $A^{(\Sigma)}$ is, at least, an algebraic space with fs $\log$ structure in the sense defined later in Section 6.2 , even if $(*)$ is not satisfied in Theorem 4.1(2). To see it, take a sufficiently large $n>0$ such that $n Y$ satisfies $(*)$ for $\Sigma$. Then, $\widetilde{A}^{(\Sigma)} / n Y$ is represented by a log smooth fs $\log$ scheme by Theorem $4.1(2)$, and the projection $\widetilde{A}^{(\Sigma)} / n Y \rightarrow A^{(\Sigma)}=$ $\left(\widetilde{A}^{(\Sigma)} / n Y\right) /(Y / n Y)$ is represented by surjective and strict étale morphisms. This shows that $A^{(\Sigma)}$ is represented by a log smooth algebraic space with fs $\log$ structure.

\section{§5. A weak log abelian variety is a két sheaf}

Here we prove, as an application of Theorem 4.1, that a weak log abelian variety is a két (Kummer log étale) sheaf. See [17] for the két topology.

THEOREM 5.1. Let $A$ be a weak log abelian variety over an fs log scheme $S$. Then, $\widetilde{A}$ and $A$ are sheaves with respect to the két topology. 
Here, when we mention $\widetilde{A}$, we assume that we are in the situation of Section 2.4 .

In the rest of this section, we prove Theorem 5.1.

Theorem 5.2. Let $Y$ be an fs log scheme over an fs log scheme $X$. Then, the functor $\operatorname{Mor}_{X}(-, Y)$ on $(\mathrm{fs} / X)$ is a sheaf for the log flat topology. In particular, it is a két sheaf.

A proof for this theorem is given in [12, Section 3], which is also included in [18, Theorem 2.20].

Here we give some supplement of that proof.

In $[12$, Sections $3.4,3.5]$, it is proved that

$$
T \mapsto \Gamma\left(T, \mathcal{O}_{T}\right) \quad \text { and } \quad T \mapsto \Gamma\left(T, M_{T}\right)
$$

are sheaves for the log flat topology, and the case of Theorem 5.2 is deduced from it where $X=\operatorname{Spec}(\mathbb{Z}), Y$ is affine, and $Y$ has a chart. It may not, however, be trivial to reduce the general case of Theorem 5.2 to this case. We now explain this step of reduction.

We may and will assume that $X=\operatorname{Spec}(\mathbb{Z})$ and that $Y$ is affine. (But we do not assume that $Y$ has a chart.) We may assume that there is an étale surjection $f: Y^{\prime} \rightarrow Y$ from an affine $Y^{\prime}$ having a chart such that the log structure of $Y^{\prime}$ is the inverse image of that of $Y$. We assume that we already know that $\operatorname{Mor}\left(-, Y^{\prime}\right)$ is a sheaf. Further, since $Y$ is affine, $\operatorname{Mor}\left(-, Y_{0}\right)$ is also a sheaf, where $Y_{0}$ is the underlying scheme of $Y$ endowed with the trivial log structure. This is seen as in [12, Section 3.3] (see [18, Theorem 2.20]); the proof there for that $F(-):=\operatorname{Mor}\left(-, Y_{0}\right)$ is a sheaf still works even when $Y$ does not have a chart.

Now let $p: U \rightarrow T$ be a covering for the logarithmic flat topology. We have to show that

$$
\operatorname{Mor}(T, Y) \rightarrow \operatorname{Mor}(U, Y) \rightrightarrows \operatorname{Mor}\left(U \times_{T} U, Y\right)
$$

is exact.

First, let $g, h$ be two morphisms from $T$ to $Y$, and assume that $g p=h p$. Then, the underlying morphisms of $g$ and $h$ coincide because $F$ is a sheaf as is noted above. Hence, the two fiber products $T^{\prime}=T \times_{Y} Y^{\prime}$ with respect to $g$ and $h$ are isomorphic over $T$ because their log structures are the inverse images of that of $T$. We identify these two $T^{\prime}$, and let $U^{\prime}$ be the fiber product $U \times_{T} T^{\prime}=U \times_{Y} Y^{\prime}$. Then the base changes $g^{\prime}$ and $h^{\prime}$ of $g$ and $h$ by $f$ coincide 
because $g^{\prime} p^{\prime}=h^{\prime} p^{\prime}$ and $\operatorname{Mor}\left(-, Y^{\prime}\right)$ is a sheaf, where $p^{\prime}$ is the base change of $p$ by $f$. Hence, $g$ and $h$ coincide.

Next, let $k$ be a morphism from $U$ to $Y$, and assume that $k p_{1}=k p_{2}$, where $p_{i}$ is the $i$ th projection $U \times_{T} U \rightarrow U$ for $i=1,2$. Similarly as above, first the underlying morphism of $k$ factors through the underlying scheme of $T$ because $F$ is a sheaf. Let $g_{0}$ be the induced morphism from the underlying scheme of $T$ to the underlying scheme of $Y$, and let $T^{\prime}$ be the fiber product of the underlying scheme of $Y^{\prime}$ and the underlying scheme of $T$ over the underlying scheme of $Y$ endowed with the inverse image of the log structure of $T$. Then, $U^{\prime}=U \times_{T} T^{\prime}$ and $U \times_{Y} Y^{\prime}$ are isomorphic over $U$, and we identify them. Again similarly as above, the base change $k^{\prime}$ of $k$ by $f$ factors through $T^{\prime}$ because $\operatorname{Mor}\left(-, Y^{\prime}\right)$ is a sheaf. Together with the uniqueness already proven, this implies that $k$ factors through $T$, which completes the proof of the exactness of the sequence $(*)$.

5.3. We prove Theorem 5.1. First we prove that $\widetilde{A}$ is a két sheaf. Consider the commutative diagram

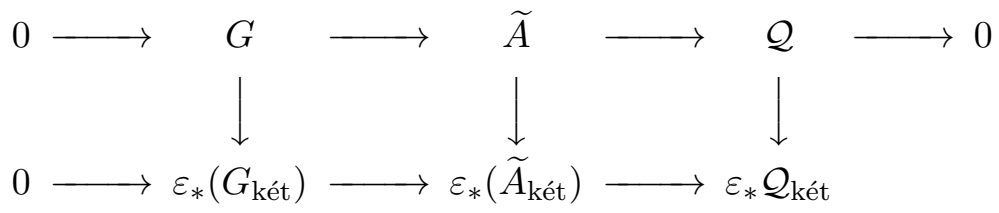

with exact rows.

Here the upper row is the last one in Section 2.4, $\varepsilon$ is the morphism of topoi from the big két site $(\mathrm{fs} / S)_{\text {két }}$ to the usual big site (fs $\left./ S\right)_{\text {ét }}$, and $0 \rightarrow G_{\text {két }} \rightarrow \widetilde{A}_{\text {két }} \rightarrow \mathcal{Q}_{\text {két }} \rightarrow 0$ is the pullback of the upper row by $\varepsilon^{-1}$.

Our task is to show that the middle vertical arrow is an isomorphism. Since the left vertical arrow is an isomorphism by Theorem 5.2 and the right vertical arrow $\mathcal{Q} \rightarrow \varepsilon_{*} \mathcal{Q}_{\text {két }} \subset \mathcal{Q} \otimes \mathbb{Q}$ is injective, we may consider the problem fiberwise. Hence, we may assume that $A$ has a constant degeneration. Moreover, we may assume that we are in the situation of Section 2.5. Then, by Theorem $4.1, \widetilde{A}^{(\Delta)}$ is represented by an fs log scheme for any finitely generated subcone $\Delta$ of $C$. Hence, it is a két sheaf by Theorem 5.2 again. Since $\widetilde{A}$ is the union of $\widetilde{A}^{(\Delta)}$, where $\Delta$ runs over the set of all finitely generated subcones of $C$, it is a két sheaf; that is, we have

$$
\varepsilon_{*}\left(\widetilde{A}_{\mathrm{két}}\right)=\widetilde{A}
$$


Next, we prove that $A$ is a két sheaf. We may assume that we are in the situation of Section 2.4. Consider the exact sequence

$$
0 \rightarrow \bar{Y} \rightarrow \widetilde{A} \rightarrow A \rightarrow 0
$$

which comes from the definition of $\widetilde{A}$ in Section 2.4. Pulling it back, we have another exact sequence

$$
0 \rightarrow \bar{Y}_{\text {két }} \rightarrow \widetilde{A}_{\text {két }} \rightarrow A_{\text {két }} \rightarrow 0 .
$$

Applying $\varepsilon_{*}$ to this sequence, and using the equalities $(*)$ and $\varepsilon_{*}\left(\bar{Y}_{\text {két }}\right)=\bar{Y}$, we have the third exact sequence

$$
0 \rightarrow \bar{Y} \rightarrow \widetilde{A} \rightarrow \varepsilon_{*}\left(A_{\text {két }}\right) \rightarrow R^{1} \varepsilon_{*} \bar{Y} .
$$

Here we also have the vanishing $R^{1} \varepsilon_{*} \bar{Y}=0$, which can be calculated fiberwise by the proper base change theorem (see [17, (5.1), (5.1.1)]). Comparing the last sequence with the original one, we see that $A=\varepsilon_{*}\left(A_{\text {két }}\right)$, that is, that $A$ is a két sheaf. This completes the proof.

\section{§6. Algebraic spaces with fs log structure}

In this section, we discuss algebraic spaces with fs log structure. An algebraic space with fs $\log$ structure is nothing but an algebraic space in the sense of M. Artin with an fs log structure on its étale site. (Recall that we can consider an fs log structure on any ringed topos.) We will explain that we can regard an algebraic space with fs log structure as a sheaf over the category of fs log schemes.

6.1. First we recall the definition of algebraic spaces in the sense of Artin. Let $S$ be a scheme. A (quasiseparated) algebraic space over $S$ is a sheaf $F$ on the category $(\operatorname{sch} / S)$ ét of schemes over $S$ endowed with the étale topology having the following properties.

6.1.1. There exists a scheme $F^{\prime}$ over $S$ and a morphism $f: F^{\prime} \rightarrow F$ over $S$ such that $f$ is represented by surjective étale morphisms of schemes.

6.1.2. The diagonal morphism $F \rightarrow F \times F$ is represented by morphisms of schemes of finite type.

Let $S$ be a scheme, and let $F$ be an algebraic space over $S$ in the above sense. Then $F$ has the small étale site $F_{\text {ét }}$. (Objects are pairs $(X, f)$, where $X$ is a scheme over $S$ and $f$ is a morphism $X \rightarrow F$ which is represented by 
étale morphisms of schemes, and the morphisms and the topology of this site are defined in the evident way.) Hence, we can discuss fs log structures on $F_{\text {ét }}$. This gives the notion of "algebraic space with fs log structure," which we will define precisely in Section 6.2 below.

6.2. Let $S$ be an fs log scheme. Recall that (fs $/ S$ ) ét denotes the category of fs log schemes over $S$ endowed with the strict étale topology.

An algebraic space with $f s$ log structure over $S$ means an algebraic space over the underlying scheme $\stackrel{\circ}{S}$ of $S$ endowed with an fs log structure over $S$.

Let $F$ be an algebraic space with fs log structure over $S$. Then $F$ defines a sheaf on $(\text { fs } / S)_{\text {ét by }} U \mapsto \operatorname{Hom}_{S}(U, F)$. (Explicitly, for any fs log scheme $U$ over $S$, the set of $U$-sections is defined to be the set of pairs $(g, h)$ of a morphism $g: \stackrel{\circ}{U} \rightarrow \stackrel{\circ}{F}$ of algebraic spaces and a homomorphism $h: g^{*} M_{F} \rightarrow$ $M_{U}$ over $M_{S}$ of log structures on $\stackrel{\circ}{U}$ ét.)

By this, we can regard the category of algebraic spaces with fs log structure over $S$ as a full subcategory of the category $(\mathrm{fs} / S)_{\text {ét }}^{\sim}$ of the sheaves

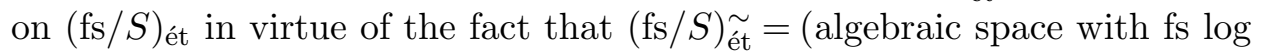
structure $/ S)_{\text {ét }}^{\sim}$. (The topology of the latter site is naturally defined.)

The full subcategory can be described as follows. To give an algebraic space with fs $\log$ structure over $S$ is equivalent to giving a sheaf $F$ on (fs $/ S)_{\text {ét }}$ satisfying the following conditions.

(1) There exist an fs $\log$ scheme $F^{\prime}$ over $S$ and a morphism $F^{\prime} \rightarrow F$ which is represented by surjective and strict étale morphisms such that the underlying morphism of schemes of the morphism $F^{\prime} \times_{F} F^{\prime} \rightarrow F^{\prime} \times \underset{S}{\circ} F^{\prime}$ of fs $\log$ schemes is a monomorphism.

(2) The diagonal morphism $F \rightarrow F \times F$ is represented by morphisms of finite type.

In fact, if we have a sheaf $F$ satisfying conditions (1) and (2), we obtain an algebraic space $J$ over $\stackrel{\circ}{S}$ and a $\log$ structure $M_{J}$ on the étale site of $J$ as follows. Let $F^{\prime} \rightarrow F$ be as in condition (1). Let $X=\stackrel{\circ}{F^{\prime}}$, and let $R=$ $\left(F^{\prime} \times{ }_{F} F^{\prime}\right)^{\circ}$. Then, $R$ is an equivalence relation on $X$ by the assumption that $R \rightarrow X \times{ }_{S}^{\circ} X$ is a monomorphism. Let $J$ be the quotient sheaf of $X$ by this equivalence relation.

We prove that $J$ is an algebraic space. Since $F^{\prime} \rightarrow F$ is represented by surjective and strict étale morphisms, the first projection $F^{\prime} \times{ }_{F} F^{\prime} \rightarrow F^{\prime}$ is represented by a surjective and strict étale morphism. Hence, its underlying morphism $R \rightarrow X$ is represented by a surjective étale morphism of schemes, 
which implies that $X \rightarrow J$ is represented by surjective étale morphisms of schemes. Next, condition (2) implies that $F^{\prime} \times{ }_{F} F^{\prime} \rightarrow F^{\prime} \times{ }_{S} F^{\prime}$ is represented by a morphism of finite type, that is, that its underlying morphism $R \rightarrow$ $\left(F^{\prime} \times{ }_{S} F^{\prime}\right)^{\circ}$ of schemes is of finite type. Since $\left(F^{\prime} \times{ }_{S} F^{\prime}\right)^{\circ} \rightarrow X \times{ }_{S} X$ is a finite morphism, the composite $R \rightarrow\left(F^{\prime} \times{ }_{S} F^{\prime}\right)^{\circ} \rightarrow X \times{ }_{S}^{\circ} X$ is a morphism of finite type. Hence, the diagonal $J \rightarrow J \times J$ is represented by morphisms of finite type, which completes the proof of the fact that $J$ is an algebraic space.

The log structure of $F^{\prime}$ with the natural descent data on $R$ gives the log structure $M_{J}$ on $J$ over $M_{S}$. The $\left(J, M_{J}\right)$ is independent of the choice of $F^{\prime} \rightarrow F$.

Conversely, for an algebraic space $J$ over $\stackrel{\circ}{S}$ with an fs $\log$ structure $M_{J}$ over $M_{S}$, we can show that the corresponding $F$ satisfies conditions (1) and (2) as follows.

First we see that the condition (1) is satisfied by taking a scheme cover $X \rightarrow J$ as in property 6.1.1, and we define $F^{\prime}$ to be the functor represented by $X$ endowed with the pullback of $M_{J}$. We prove condition (2). Since the composite $J \stackrel{d}{\rightarrow}\left(\left(J, M_{J}\right) \times_{S}\left(J, M_{J}\right)\right)^{\circ} \stackrel{q}{\rightarrow} J \times_{S^{\circ}} J$ is quasicompact as a morphism of algebraic spaces and $q$ is finite, the morphism $d$ of algebraic spaces is quasicompact. It follows that $F \rightarrow F \times{ }_{S} F$ is represented by morphisms of finite type.

\section{$\S 7$. Log Artin's criterion}

In [1, Theorem 3.4], M. Artin obtained a criterion for a functor to be an algebraic space. To prove the representability of models in general, we use a log version of Artin's criterion. We remark that M. C. Olsson studied another log version of Artin's criterion in [19, Section 3.5].

7.1. A contravariant functor $F:(\mathrm{fs} / S) \rightarrow$ (set) is said to be locally of finite presentation if the following condition is satisfied: if $U$ is an fs $\log$ scheme over $S$ whose underlying scheme is an affine scheme $\operatorname{Spec} R$, and if $\left(R_{\lambda}\right)_{\lambda}$ is a filtered direct system of rings over $R$, we have

$$
\lim _{\longrightarrow} F\left(\operatorname{Spec}\left(R_{\lambda}\right)\right) \stackrel{\cong}{\longrightarrow} F\left(\operatorname{Spec}\left(\lim _{\longrightarrow} R_{\lambda}\right)\right)
$$

where $\operatorname{Spec}\left(R_{\lambda}\right)$ and $\operatorname{Spec}\left(\lim _{\longrightarrow} R_{\lambda}\right)$ are endowed with the inverse images of the $\log$ structure of $U$. 
For example, the functor $\mathbb{G}_{m, \log }$ and $U \mapsto \Gamma\left(U, M_{U}\right)$ are locally of finite presentation. The latter implies that, for an fs log scheme $X$ over $S, X$ is locally of finite presentation in the above sense if and only if the underlying scheme of $X$ is locally of finite presentation over that of $S$ in the usual sense.

Proposition 7.2. Let $S$ be an fs log scheme whose underlying scheme is of finite type over a field or over an excellent Dedekind domain. Let $F$ be a

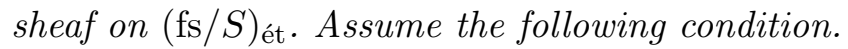

7.2.1. The diagonal morphism $F \rightarrow F \times F$ is represented by morphisms of finite type.

Consider the following condition.

(*) There exist an $f$ s log scheme $F^{\prime}$ over $S$ and a morphism $F^{\prime} \rightarrow F$ which is represented by surjective and strict étale morphisms.

Then, condition $(*)$ is satisfied if and only if $F$ satisfies the following conditions.

7.2.2. Finiteness. The sheaf $F$ is locally of finite presentation (see Section 7.1 ).

7.2.3. Effective pro-representability. The sheaf $F$ is effectively prorepresentable in the sense explained in Definition 7.3 below.

7.2.4. Openness of versality. Let $U$ be an $f$ s log scheme over $S$ of finite type, let $f: U \rightarrow F$ be a morphism, and let $u \in U$. Assume that $f$ is formally strict étale at $u \in U$ in the sense in Definition $\% .4$ below. Then $f$ is formally strict étale at each point of some neighborhood of $u$ in $U$.

Definition 7.3. For a sheaf $F$ on $(\mathrm{fs} / S)_{\text {ét }}$, we say that $F$ is effectively pro-representable if there exist a family of triples $\left(W_{\lambda}, w_{\lambda}, \xi_{\lambda}\right)(\lambda \in \Lambda)$ of fs $\log$ schemes $W_{\lambda}$ over $S$ of finite type, points $w_{\lambda}$ of $W_{\lambda}$, and morphisms $\xi_{\lambda}: \operatorname{Spec}\left(\hat{\mathcal{O}}_{W_{\lambda}, w_{\lambda}}\right) \rightarrow F$ satisfying the following condition: for an fs log scheme $U$ over $S$ such that the underlying scheme of $U$ is the spectrum of a local Artin ring, and a morphism $f: U \rightarrow F$, there exist a unique index $\lambda$ and a unique morphism $g: U \rightarrow \operatorname{Spec}\left(\hat{\mathcal{O}}_{W_{\lambda}, w_{\lambda}}\right)$ whose image is $w_{\lambda}$ such that $\xi_{\lambda} \circ g=f$. Here $\operatorname{Spec}\left(\hat{\mathcal{O}}_{W_{\lambda}, w_{\lambda}}\right)$ is endowed with the inverse image of the $\log$ structure of $W_{\lambda}$.

Definition 7.4 (see [1, Definition 3.1]). Let $S$ be an fs log scheme, let $F$ be a sheaf on $(\mathrm{fs} / S)_{\text {ét }}$, let $U$ be an fs log scheme over $S$, let $f: U \rightarrow F$ 
be a morphism, and let $u \in U$. We say that $f$ is formally strict étale (resp., formally log étale, formally strict) at $u \in U$ if for any commutative diagram

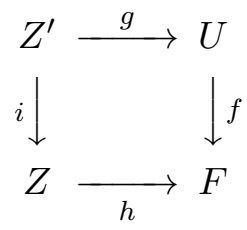

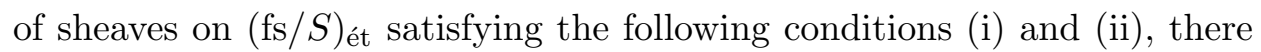
exists a unique morphism $k: Z \rightarrow U$ such that $g=k \circ i$ and $h=f \circ k$ :

(i) The sheaves $Z$ and $Z^{\prime}$ are represented by fs log schemes over $S$ whose underlying schemes are spectra of local Artinian rings, and the image of the underlying morphism of schemes of $g$ is $u$; and

(ii) the underlying morphism of schemes of $i$ is a closed immersion (resp., the morphism $i$ is a strict closed immersion, resp., the underlying morphism of schemes of $i$ is an isomorphism).

REMARK 7.4.1. As is easily seen, a morphism is formally strict étale at $u$ if and only if it is formally log étale at $u$ and formally strict at $u$.

\subsection{Proof of Proposition 7.2}

Proof. The proof is essentially the same as that of the nonlog version given by Artin [1, Theorem 3.4].

First, we show that condition $(*)$ implies conditions 7.2.2-7.2.4.

We show condition 7.2.2. Considering the cover $F^{\prime}$ in condition $(*)$ and the equivalence relation $F^{\prime} \times{ }_{F} F^{\prime}$, we see that condition 7.2.2 is reduced to the fact that the functor associated with an fs log scheme locally of finite presentation over $S$ is locally of finite presentation, which is mentioned in the last paragraph in Section 7.1.

The remaining conditions 7.2.3 and 7.2.4 are easily verified by using nonlog statements [2, Theorem 2.4] and [1, Lemma 3.3], respectively.

Next, we prove that condition $(*)$ holds for any sheaf $F$ satisfying conditions 7.2.1-7.2.4. By condition 7.2.3, there is a family of triples $\left(W_{\lambda}, w_{\lambda}, \xi_{\lambda}\right)$ as in Definition 7.3. For $\lambda \in \Lambda$, let $\mathcal{O}_{W_{\lambda}, w_{\lambda}}^{h} \subset \hat{\mathcal{O}}_{W_{\lambda}, w_{\lambda}}$ be the Henselization of the local ring $\left(\mathcal{O}_{W_{\lambda}, w_{\lambda}}, m_{w_{\lambda}}\right)$. By condition 7.2 .2 , there is a finitely generated subring $R$ of $\hat{\mathcal{O}}_{W_{\lambda}, w_{\lambda}}$ over $\mathcal{O}_{W_{\lambda}, w_{\lambda}}^{h}$ such that $\xi_{\lambda}$ comes from an element $\xi_{\lambda}^{\prime}$ of $F(\operatorname{Spec}(R))$. By Artin's approximation theorem, there is a homomorphism $s: R \rightarrow \mathcal{O}_{W_{\lambda}, w_{\lambda}}^{h}$ over $\mathcal{O}_{W_{\lambda}, w_{\lambda}}^{h}$ such that the composite $R \stackrel{s}{\rightarrow} \mathcal{O}_{W_{\lambda}, w_{\lambda}}^{h} \rightarrow$ $\mathcal{O}_{W_{\lambda}, w_{\lambda}} / m_{w_{\lambda}}^{2}$ coincides with the composite $R \stackrel{\subseteq}{\rightarrow} \hat{\mathcal{O}}_{W_{\lambda}, w_{\lambda}} \rightarrow \mathcal{O}_{W_{\lambda}, w_{\lambda}} / m_{w_{\lambda}}^{2}$. 
Let $\xi_{\lambda}^{\prime \prime} \in F\left(\operatorname{Spec}\left(\mathcal{O}_{W_{\lambda}, w_{\lambda}}^{h}\right)\right)$ be the image of $\xi_{\lambda}^{\prime}$ under $s$. By the property of $\left(W_{\lambda}, w_{\lambda}, \xi_{\lambda}\right)$ in condition 7.2 .3 , for any $n \geq 0$ there exists a unique morphism $g_{\lambda, n}: \operatorname{Spec}\left(\mathcal{O}_{W_{\lambda}, w_{\lambda}} / m_{w_{\lambda}}^{n+1}\right) \rightarrow \operatorname{Spec}\left(\hat{\mathcal{O}}_{W_{\lambda}, w_{\lambda}}\right)$ over $S$ whose composite with $\xi_{\lambda}$ coincides with the composite $\operatorname{Spec}\left(\mathcal{O}_{W_{\lambda}, w_{\lambda}} / m_{w_{\lambda}}^{n+1}\right) \rightarrow \operatorname{Spec}\left(\mathcal{O}_{W_{\lambda}, w_{\lambda}}^{h}\right) \stackrel{\xi_{\lambda}^{\prime \prime}}{\rightarrow}$ $F$. Note that, by the uniqueness, $g_{\lambda, 1}$ coincides with the canonical inclusion. The limit of $g_{\lambda, n}$ for $n \rightarrow \infty$ gives a morphism $g_{\lambda}: \operatorname{Spec}\left(\hat{\mathcal{O}}_{W_{\lambda}, w_{\lambda}}\right) \rightarrow$ $\operatorname{Spec}\left(\hat{\mathcal{O}}_{W_{\lambda}, w_{\lambda}}\right)$. Since $g_{\lambda, 1}$ coincides with the inclusion morphism, $g_{\lambda}$ is an automorphism of $\operatorname{Spec}\left(\hat{\mathcal{O}}_{W_{\lambda}, w_{\lambda}}\right)$. Let $\beta_{\lambda}: \operatorname{Spec}\left(\hat{\mathcal{O}}_{W_{\lambda}, w_{\lambda}}\right) \rightarrow \operatorname{Spec}\left(\mathcal{O}_{W_{\lambda}, w_{\lambda}}^{h}\right)$ be the composite of $g_{\lambda}^{-1}$ and the canonical morphism $\operatorname{Spec}\left(\hat{\mathcal{O}}_{W_{\lambda}, w_{\lambda}}\right) \rightarrow$ $\operatorname{Spec}\left(\mathcal{O}_{W_{\lambda}, w_{\lambda}}^{h}\right)$. Then the pullback of $\xi_{\lambda}^{\prime \prime}$ under $\beta_{\lambda}$ coincides with $\xi_{\lambda}$. By the condition 7.2.2 of $F, \xi_{\lambda}^{\prime \prime}$ comes from an étale neighborhood $W_{\lambda}^{\prime} \rightarrow W_{\lambda}$ of $w_{\lambda}$. Since $\operatorname{Spec}\left(\hat{\mathcal{O}}_{W_{\lambda}, w_{\lambda}}\right) \rightarrow F$ is formally strict étale at $w_{\lambda}$ by condition 7.2 .3 , $W_{\lambda}^{\prime} \rightarrow F$ is formally strict étale at $w_{\lambda}$. Hence, $W_{\lambda}^{\prime} \rightarrow F$ is formally strict étale at each point of an open neighborhood $W_{\lambda}^{\prime \prime}$ of $w_{\lambda}$ in $W_{\lambda}^{\prime}$ by condition 7.2.4. Let $F^{\prime}$ be the disjoint union of $W_{\lambda}^{\prime \prime}$ for $\lambda \in \Lambda$, and let $F^{\prime} \rightarrow F$ be the canonical morphism. It is enough to show that $F^{\prime} \rightarrow F$ satisfies condition (*). Let $V$ be any fs $\log$ scheme over $S$, and let $V \rightarrow F$ be any morphism over $S$. Let $f$ be the base change $V^{\prime}:=V \times_{F} F^{\prime} \rightarrow V$ of $F^{\prime} \rightarrow F$. Then, $f$ is formally strict étale, as $F^{\prime} \rightarrow F$ is so. Since $F^{\prime}$ is locally of finite presentation over $S$ and since $F$ is locally of finite type over $S$, the morphism $f$ is also locally of finite presentation. Thus, $f$ is strict étale. Further, let $v$ be any fs log point, and let $v \rightarrow V$ be any morphism. By condition 7.2.3, there is a $\lambda$ such that $v \rightarrow V \rightarrow F$ factors through $w_{\lambda} \rightarrow \operatorname{Spec}\left(\hat{\mathcal{O}}_{W_{\lambda}, w_{\lambda}}\right) \rightarrow F$, factors through $W_{\lambda}^{\prime \prime}$, and hence, factors through $F^{\prime}$. Therefore, $v \rightarrow V$ factors through $V^{\prime}$, which implies the surjectivity of $f$. Thus, $F^{\prime} \rightarrow F$ satisfies condition $(*)$.

Remark 7.6. Proposition 7.2 is weaker than [1, Theorem 3.4] in the nonlog case, for we modified the effective pro-representability. In [1], the existence of $W_{\lambda}$ of finite type over $S$ is not assumed in the effective prorepresentability.

\section{$\S 8$. Models}

In this section, we start to show the general case (Theorem 8.1) of the theorem that a model of a weak log abelian variety is an algebraic space with fs log structure. A special case was proved in Theorem 4.1. 
TheOREM 8.1. Let $S$ be an fs log scheme, and let $A$ be a weak log abelian variety over $S$. Assume that there are an exact sequence

$$
0 \rightarrow G \rightarrow A \rightarrow \mathcal{H} \operatorname{om}\left(X, \mathbb{G}_{m, \log } / \mathbb{G}_{m}\right)^{(Y)} / \bar{Y} \rightarrow 0
$$

a homomorphism $\mathcal{S} \rightarrow M_{S} / \mathcal{O}_{S}^{\times}$, and an admissible pairing $X \times Y \rightarrow \mathcal{S}^{\mathrm{gp}}$ as in Sections 2.4 and 2.5. Let $C \subset \operatorname{Hom}(\mathcal{S}, \mathbb{N}) \times \operatorname{Hom}(X, \mathbb{Z})$ be as in Section 2.2, and let $\Sigma$ be a fan in $C$. Then we have the following.

(1) The $\Sigma$-part $\widetilde{A}^{(\Sigma)}$ of $\widetilde{A}$ (see Section 2.6) is an algebraic space with fs log structure over $S$.

(2) If $\Sigma$ is stable under the action of $Y$, then $A^{(\Sigma)}$ is an algebraic space with fs log structure over $S$.

\section{REMARKS.}

(1) Recall that $y \in Y$ acts on $C$ by $(N, l) \mapsto(N, l+N(\langle-, y\rangle))$.

(2) The support of $\Sigma$ need not cover $C$.

(3) Both $\widetilde{A}^{(\Sigma)}$ and $A^{(\Sigma)}$ over $S$ are $\log$ smooth in the following sense: an algebraic space $F$ with fs $\log$ structure over $S$ is $\log$ smooth if there is a $\log$ smooth fs log scheme $F^{\prime}$ over $S$ which covers $F$ like in Section 6.2, condition (1). This is seen by using the proof of Theorem 8.1 below together with Theorem 4.1 .

(4) Definition 1.6(3) is not necessary; that is, the conclusion of Theorem 8.1 still holds for a sheaf of abelian groups on (fs $/ S$ ) ét satisfying only Definition 1.6(1), (2). (The definition of $\Sigma$-part for such a sheaf is the same.) We use the case of this fact where the base $S$ is of finite type over $\mathbb{Z}$ later in Section 9.

In the rest of this section, we prove Theorem 8.1 in the case where $S$ is of finite type over a field or over an excellent Dedekind domain. After preparations in Section 9, we prove the general case in Section 9.13.

8.2. We assume that $S$ is of finite type over a field or over an excellent Dedekind domain. Let $A$ be a sheaf of abelian groups on (fs $/ S$ ) ét satisfying Definition 1.6(1), (2). We prove Theorem 8.1(1), (2) for $A$ (see Theorem 8.1, Remark (4)). The proofs of (1) and (2) are similar, so we give only the proof of Theorem 8.1(1). In Sections 8.3-8.6, we check that $\widetilde{A}^{(\Sigma)}$ satisfies conditions 7.2.1-7.2.4, respectively. Let

$$
\mathcal{Q}=\mathcal{H} o m\left(X, \mathbb{G}_{m, \log } / \mathbb{G}_{m}\right)^{(Y)}=\widetilde{A} / G
$$


and let

$$
\mathcal{Q}^{(\Sigma)}=(\text { the } \Sigma \text {-part of } \mathcal{Q})=\widetilde{A}^{(\Sigma)} / G
$$

(In the proof for $A^{(\Sigma)}$, we replace $\mathcal{Q}$ and $\mathcal{Q}^{(\Sigma)}$ by $\mathcal{Q} / \bar{Y}$ and $\mathcal{Q}^{(\Sigma)} / \bar{Y}$, respectively.)

8.3. The fact that $\widetilde{A}^{(\Sigma)} \rightarrow \widetilde{A}^{(\Sigma)} \times \widetilde{A}^{(\Sigma)}$ is represented by morphisms of finite type is reduced to the fact that $\widetilde{A}$ has the same property. The latter is equivalent to that the zero section $S \rightarrow \widetilde{A}$ is represented by morphisms of finite type and is equivalent to the fact that $\mathcal{Q}$ and $G$ have the same properties.

8.4. The fact that $\widetilde{A}^{(\Sigma)}$ is locally of finite presentation is reduced to the fact that both $\mathcal{Q}^{(\Sigma)}$ and $G$ are locally of finite presentation.

8.5. We prove that $\widetilde{A}^{(\Sigma)}$ satisfies the effective pro-representability. This is a consequence of Theorem 4.1. In fact, by Theorem 4.1, for any subscheme $S^{\prime}$ of $S$ such that $M_{S^{\prime}} / \mathcal{O}_{S^{\prime}}^{\times}$is locally constant, the pullback of $\widetilde{A}^{(\Sigma)}$ to (fs $/ S^{\prime}$ ) is represented by a log smooth fs log scheme over $S^{\prime}$. Hence, for each $s \in S$, the pullback of $\widetilde{A}^{(\Sigma)}$ to $\left(\mathrm{fs} / \hat{S}_{s}\right)$ is represented by a log smooth formal fs $\log$ scheme $A_{s}$ over the formal completion $\hat{S}_{s}$ of $S$ at $s$ (endowed with the pullback $\log$ structure from $S$ ). Let $\Lambda$ be the set-theoretic disjoint union of the special fibers of all such $A_{s}(s \in S)$. For each $\lambda \in \Lambda$, let $\xi_{\lambda}: \operatorname{Spec}\left(R_{\lambda}\right) \rightarrow$ $A_{s} \rightarrow F$ be the canonical morphism from the completed local ring of $A_{s}$ at $\lambda$ (endowed with the pullback $\log$ structure from $A_{s}$ ). Since $A_{s}$ is $\log$ smooth over $\hat{S}_{s}$, the fs log scheme $\operatorname{Spec}\left(R_{\lambda}\right)$ is algebraizable; that is, there is an fs $\log$ scheme $W_{\lambda}$ over $S$ of finite type and a point $w_{\lambda} \in W_{\lambda}$ such that $R_{\lambda}$ is isomorphic to $\hat{\mathcal{O}}_{W_{\lambda}, w_{\lambda}}$. These triples $\left(W_{\lambda}, w_{\lambda}, \xi_{\lambda}\right)(\lambda \in \Lambda)$ form the desired family.

8.6. We prove that $\widetilde{A}^{(\Sigma)}$ satisfies the openness of versality. Let $U$ be an fs $\log$ scheme over $S$ of finite type, let $f: U \rightarrow \widetilde{A}^{(\Sigma)}$ be a morphism, and let $u \in U$. Assume that $f$ is formally strict étale at $u$. We prove that $f$ is formally strict étale at each point of some open neighborhood of $u$. There exist an fs log scheme $V$ over $S$ and a morphism $V \rightarrow \mathcal{Q}^{(\Sigma)}$ which is represented by surjective and strict smooth morphisms. Let $f^{\prime}$ be the base change $U \times_{\mathcal{Q}^{(\Sigma)}} V \rightarrow \widetilde{A}^{(\Sigma)} \times_{\mathcal{Q}^{(\Sigma)}} V$ of $f$, and let $u^{\prime}$ be a point of the scheme $U \times{ }_{\mathcal{Q}^{(\Sigma)}} V$ lying over $u$. Then $f^{\prime}$ is formally strict étale at $u^{\prime}$. Since $f^{\prime}$ is a morphism of fs log schemes and is of finite type, $f^{\prime}$ is strict étale on some open neighborhood $U^{\prime}$ of $u^{\prime}$ in $U \times_{\mathcal{Q}^{(\Sigma)}} V$. Let $U^{\prime \prime}$ be the image of $U^{\prime}$ in $U$. 
Since $U \times{ }_{\mathcal{Q}^{(\Sigma)}} V \rightarrow U$ is strict smooth and hence is an open map, $U^{\prime \prime}$ is an open neighborhood of $u$ in $U$. It is easily seen that $f$ is formally strict étale at each point of $U^{\prime \prime}$.

8.7. Now we have checked all the necessary conditions to apply Proposition 7.2. Hence, by Proposition 7.2, there is an fs log scheme $F^{\prime}=\left(X, M_{X}\right)$ and a morphism $F^{\prime} \rightarrow \widetilde{A}^{(\Sigma)}$ which is represented by surjective and strict étale morphisms. To complete the proof of Theorem 8.1 in the present case, it is enough to prove that $i: R \rightarrow X \times X$ is a monomorphism, where $R$ is the underlying scheme of $F^{\prime} \times_{\widetilde{A}^{(\Sigma)}} F^{\prime}$. By construction in the proof of Proposition $7.2, F^{\prime}$ can be pulled back by any strict immersion $S^{\prime} \rightarrow S$ so that $X$ and $R$ can be pulled back, too.

Let $T$ be a test scheme over $\stackrel{\circ}{S}$. Let $f, g: T \rightarrow R$ be two morphisms such that $i \circ f=i \circ g$. It is enough to show that $f=g$. First, if $S$ has a constant log structure, then $\widetilde{A}^{(\Sigma)}$ is represented by an fs log scheme (see Theorem 4.1) and $f=g$. Next, by considering the base change by the projection from the disjoint union of the constant log loci (i.e., the strata where the log is locally constant) of $S$ to $S$, we see that the underlying maps of sets of $f$ and $g$ coincide. Then, we may and will assume that $T$ is affine.

If, further, $T$ is Noetherian, then the projection from the disjoint union of all constant log loci of $T$ (including thickenings) to $T$ is an epimorphism (by the fact that for any element $a$ of a Noetherian ring $A$, the homomorphism $A \rightarrow A_{a} \times \hat{A}$ is injective). Hence, $f$ and $g$ coincide, as desired.

The general case reduces to the previous case where $T$ is Noetherian because $X$ and $R$ are locally Noetherian. In conclusion, we have $f=g$ in general, and $i$ is certainly a monomorphism, which completes the proof of Theorem 8.1(1) in the present case that $\widetilde{A}^{(\Sigma)}$ is an algebraic space with fs $\log$ structure over $S$.

The following results will be used later in Proposition 12.7. Their proofs are easy once Theorem 8.1 is proved.

Proposition 8.8. Let $A$ be a weak log abelian variety over an $f s$ log scheme S. Assume that we are in the situation of Sections 2.4 and 2.5. Let $\Sigma$ be a fan which is stable under the action of $Y$.

(1) Let $\Sigma^{\prime}$ be a subdivision of $\Sigma$ which is also $Y$-stable. Then the natural morphism $A^{\left(\Sigma^{\prime}\right)} \rightarrow A^{(\Sigma)}$ is proper and surjective.

(2) Let $\Sigma^{\prime}$ be a subfan of $\Sigma$ which is also $Y$-stable. Then the natural morphism $A^{\left(\Sigma^{\prime}\right)} \rightarrow A^{(\Sigma)}$ is a strict open immersion. 


\section{$\S 9$. Weak log abelian varieties over limits}

In this section, we prove that the category of weak log abelian varieties over a filtered limit of quasicompact and quasiseparated fs log schemes $S_{\lambda}$ is equivalent to the limit of the categories of weak log abelian varieties over $S_{\lambda}$ (see [10, Section 5.3(1)] for the 1-dimensional (log elliptic curve) case). This result is fundamental and will be repeatedly used in future parts in our series. As an application, in the end of this section, we use this to complete the proof of Theorem 8.1.

9.1. For an fs log scheme $T$, let $F(T)$ be the set of isomorphism classes of weak log abelian varieties over $T$.

Let $\left(S_{\lambda}\right)_{\lambda}$ be a filtered projective system of quasicompact and quasiseparated fs log schemes whose transition morphisms are affine and strict. Then, $S:=\lim _{\longleftarrow} S_{\lambda}$ is represented by an fs log scheme whose underlying scheme is the projective limit of the underlying schemes of $S_{\lambda}$ and whose log structure is the inverse image log structure of that of any $S_{\lambda}$.

Proposition 9.2. Let $S:=\lim _{\longleftarrow} S_{\lambda}$ be as above.

(1) Let $A_{1}, A_{2}$ be weak log abelian varieties over some $S_{\lambda_{0}}$. Then, the natural map

$$
\underset{\lim }{\longrightarrow} \operatorname{Hom}_{S_{\lambda}}\left(\left(A_{1}\right)_{S_{\lambda}},\left(A_{2}\right)_{S_{\lambda}}\right) \rightarrow \operatorname{Hom}_{S}\left(\left(A_{1}\right)_{S},\left(A_{2}\right)_{S}\right)
$$

is bijective. Here, $\left(A_{i}\right)_{S_{\lambda}}$ and $\left(A_{i}\right)_{S}$ are the pullbacks of $A_{i}$ to $S_{\lambda}$ and $S$, respectively $(i=1,2)$. In particular, if $\left(A_{1}\right)_{S}$ and $\left(A_{2}\right)_{S}$ are isomorphic over $S$, then there is some $\lambda_{0} \rightarrow \lambda$ such that $\left(A_{1}\right)_{S_{\lambda}}$ and $\left(A_{2}\right)_{S_{\lambda}}$ are already isomorphic over $S_{\lambda}$.

(2) The natural map

$$
\lim _{\longrightarrow} F\left(S_{\lambda}\right) \rightarrow F(S)
$$

is bijective. In particular, for a weak log abelian variety $A$ over $S$, there are an index $\lambda$ and a weak log abelian variety $A_{\lambda}$ over $S_{\lambda}$ whose pullback to $S$ is isomorphic to $A$.

We prove this proposition in 9.6-9.12.

Let $(\mathrm{WLAV} / S)$ be the category of weak log abelian varieties over an fs $\log$ scheme $S$.

Corollary 9.3. As a category, we have $(\mathrm{WLAV} / S)=\underset{\longrightarrow}{\lim }\left(\mathrm{WLAV} / S_{\lambda}\right)$. 
Corollary 9.4. The functor $F:(\mathrm{fs} / S) \rightarrow($ set $)$ in Section 9.1 is locally of finite presentation in the sense of Section \%.1.

Corollary 9.5. Any weak log abelian variety over an affine scheme is defined over a finitely generated ring over $\mathbb{Z}$.

9.6. To prove Proposition 9.2, we may assume that there is an initial object in the index category. In Proposition 9.2(1), we may assume that it is $\lambda_{0}$. In Proposition 9.2(2), we denote it by $\lambda_{0}$. In general, Proposition 9.2(1) implies the injectivity of the map in Proposition 9.2(2). Let $\left(S_{\lambda_{0}, j} \rightarrow S_{\lambda_{0}}\right)_{j \in J}$ be the strict étale covering with a finite index set $J$. Then, if Proposition 9.2(1) holds for the objects obtained by base change with respect to $S_{\lambda_{0}, j} \rightarrow S_{\lambda_{0}}$ for each $j \in J$, it holds for the original one. Further, Proposition 9.2(1) implies that the surjectivity of Proposition 9.2(2) reduces to the case of the objects obtained by base change with respect to $S_{\lambda_{0}, j} \rightarrow S_{\lambda_{0}}$ for each $j$. (Here we use the easy fact that an abelian sheaf on the big site of an fs log scheme is a weak log abelian variety if and only if so is it strict étale locally.) Thus, in proving Proposition 9.2, we may assume that there is a prime $\ell$ such that $\ell$ is invertible on $S_{\lambda_{0}}$. In the following, we will assume that there is such an $\ell$ and fix it.

Further, it is enough to prove the following statements $(1)^{\prime}$ and $(2)^{\prime}$. Let the situation be as in Proposition 9.2.

(1)' Proposition 9.2(1) holds if there are a homomorphism from an fs monoid $\mathcal{S} \rightarrow M_{S_{\lambda_{0}}} / \mathcal{O}_{S_{\lambda_{0}}}^{\times}$, admissible pairings $X_{i} \times Y_{i} \rightarrow \mathcal{S}^{\text {gp }}$, and exact sequences $0 \rightarrow G_{i} \rightarrow A_{i} \rightarrow \mathcal{Q}_{i} / \bar{Y}_{i} \rightarrow 0$ as in Sections 2.4 and $2.5(i=1,2)$.

$(2)^{\prime}$ Let $A$ be a weak $\log$ abelian variety $A$ over $S$. Assume that there are a homomorphism from an fs monoid $\mathcal{S} \rightarrow M_{S} / \mathcal{O}_{S}^{\times}$, an admissible pairing $X \times$ $Y \rightarrow \mathcal{S}^{\mathrm{gp}}$, and an exact sequence $0 \rightarrow G \rightarrow A \rightarrow \mathcal{Q} / \bar{Y} \rightarrow 0$ as in Sections 2.4 and 2.5. Then, there are an index $\lambda$ and a weak log abelian variety $A_{\lambda}$ over $S_{\lambda}$ whose pullback to $S$ is isomorphic to $A$.

9.7. The proofs for $(1)^{\prime}$ and $(2)^{\prime}$ in Section 9.6 are similar, and we describe only the proof for $(2)^{\prime}$ after proving some propositions.

First, let $A$ be a weak log abelian variety over an fs log scheme $S$. Assume that there are a homomorphism from an fs monoid $\mathcal{S} \rightarrow M_{S} / \mathcal{O}_{S}^{\times}$, an admissible pairing $X \times Y \rightarrow \mathcal{S}^{\mathrm{gp}}$, and an exact sequence $0 \rightarrow G \rightarrow A \rightarrow \mathcal{Q} / \bar{Y} \rightarrow 0$ as in Sections 2.4-2.5. Then, we have an exact sequence $0 \rightarrow G \rightarrow \widetilde{A} \rightarrow \mathcal{Q} \rightarrow 0$ as in Section 2.4. Assume that there is a prime number $\ell$ which is invertible on the base. 
Take a finitely generated subcone $\sigma$ of $C$ (in the terminology of Section 2.2) such that $\bigcup_{n} \ell^{n} \sigma=C$, where $\ell^{n} \sigma$ is defined in Section 2.2. (Such a cone exists. Indeed, the cone $C(1)$ in [8, Section 3.4.9] satisfies the condition, as is seen in [8, Lemma 3.4.10].) Then, we have $\bigcup_{n}\left[\ell^{n} \sigma\right]=\mathcal{Q}$ (see Section 2.3 for the notation $[-])$. Let $I:=\widetilde{A}^{(\sigma)} \subset \widetilde{A}$ be the part corresponding to $\sigma$, that is, the pullback of $[\sigma]$. Then $I$ is a $G$-torsor over $[\sigma]$ with respect to the étale topology. Let $J=\widetilde{A}^{\left(\ell^{-1} \sigma\right)} \subset I$. We have a surjection of két sheaves $J \rightarrow I ; x \mapsto x^{\ell}$ (see Theorem 5.1).

Proposition 9.8. Consider the két sheaf of sets $\widetilde{L}=\{(x, n) \mid x \in I, n \geq$ $0\} / \sim$, where $\sim$ is the equivalence relation generated by the following.

(1) If $x \in J$, then $\left(x^{\ell}, n\right) \sim(x, n+1)$ for $n \geq 0$.

(2) If $a \in G, x \in I, n \geq 0$, and $a^{\ell^{n}}=1$, then $(a x, n) \sim(x, n)$.

Then we have an isomorphism of két sheaves

$$
\widetilde{L} \cong \widetilde{A} ; \quad(x, n) \mapsto x^{\ell^{n}} .
$$

Proof. The well-definedness of the map is by condition (1). By the property $\bigcup_{n} \ell^{n} \sigma=C$ of $\sigma$, the map is surjective. The injectivity is proved by condition (2).

9.9. The group structure of $\widetilde{L}$ (written multiplicatively) corresponding to the group structure of $\widetilde{A}$ is characterized by the property $(x, n)(y, n)=$ $(x y, n)$ for $x, y \in J$ (note that $x y \in I)$.

LEMMA 9.10. Let the notation and the assumption be as in Sections 2.1 and 2.2. Let $\sigma$ be a finitely generated subcone of $C$ such that $\sigma$ maps onto $\mathcal{S}^{\vee}$. Let $Z$ be a scheme of finite type over $S$. Then, any morphism $f:[\sigma] \rightarrow Z$ is constant.

REMARK. The surjectivity assumption on $\sigma \rightarrow \mathcal{S}^{\vee}$ is necessary. In fact, consider the case where $X$ is trivial. Then $[\sigma]$ is a partial log blowing up of the base. The identity morphism from it to itself is not constant unless it coincides with the base.

Proof. We may assume that the base $S$ is affine, say, $\operatorname{Spec} R$. By the assumption, we see that the homomorphism $\mathcal{S} \rightarrow \sigma^{\vee}$ is exact. Since $[\sigma]=$ $V(\sigma) / \mathcal{H o m}\left(X, \mathbb{G}_{m}\right)$, the given $f$ induces a morphism $V(\sigma) \rightarrow Z$ of schemes. Let $z \in Z$ be the image of the closed orbit of $V(\sigma)$. Then, $f$ factors into any open neighborhood of $z$. Hence, we may assume that $Z$ is also affine. 
Further, we may assume that $Z$ is the affine line over $S$. Again, since $[\sigma]=V(\sigma) / \mathcal{H o m}\left(X, \mathbb{G}_{m}\right)$, the lemma is reduced to the following: if an element $a$ of the coordinate ring $U:=R \otimes_{\mathbb{Z}[\mathcal{S}]} \mathbb{Z}\left[\sigma^{\vee}\right]$ of $V(\sigma)$ is $\operatorname{Hom}\left(X, \mathbb{G}_{m}\right)$ invariant, it belongs to $R$. Here the $\operatorname{Hom}\left(X, \mathbb{G}_{m}\right)$-invariance implies that, for any ring homomorphism $R \rightarrow R^{\prime}$, the image of $a$ in $R^{\prime} \otimes_{R} U$ is $\operatorname{Hom}\left(X, R^{\prime \times}\right)$ invariant. Thus, by strictly localizing $R$, we may assume that $R$ satisfies the following condition.

(*) For any $n>0$, there is an invertible element $r$ of $R$ such that $1-r^{n}$ is also invertible.

Below, we assume this. Let $a$ be an element of $U$, and assume that $a$ is $\operatorname{Hom}\left(X, R^{\times}\right)$-invariant. We will show that $a \in R$. For each $x \in X$, consider the $R$-submodule $U_{x}$ of $U$ generated by the set $\left\{x \mathcal{S}^{\mathrm{gp}} \cap \sigma^{\vee}\right\}$. Then, $U$ is the direct sum decomposition of $U_{x}$. Further, by virtue of condition $(*)$, $U_{x}$ is characterized as the part of $U$ on which the group $\operatorname{Hom}\left(X, R^{\times}\right)$acts via $x$. Hence, $a$ belongs to $U_{0}$, which coincides with $R$ by the exactness $\mathcal{S}^{\mathrm{gp}} \cap \sigma^{\vee}=\mathcal{S}$.

LEMMA 9.11. Let the notation and the assumption be as in Sections 2.1 and 2.2. Let $\mathcal{S}^{\prime} \rightarrow M_{S} / \mathcal{O}_{S}^{\times}$be another homomorphism from an fs monoid, and let $\langle,\rangle^{\prime}: X^{\prime} \times Y^{\prime} \rightarrow \mathcal{S}^{\prime g p}$ be another admissible pairing. Let $\mathcal{Q}^{\prime}$ be the associated sheaf of abelian groups as in Section 1.3, and let $C^{\prime}$ be the associated cone as in Section 2.2.

Let $X^{\prime} \rightarrow X, Y \rightarrow Y^{\prime}$, and $\mathcal{S}^{\prime} \rightarrow \mathcal{S}$ be homomorphisms which are compatible with $\langle\rangle,,\langle,\rangle^{\prime}, \mathcal{S} \rightarrow M_{S} / \mathcal{O}_{S}^{\times}$, and $\mathcal{S}^{\prime} \rightarrow M_{S} / \mathcal{O}_{S}^{\times}$. Let $h$ be the induced homomorphism $\mathcal{Q} \rightarrow \mathcal{Q}^{\prime}$.

Let $\sigma$ (resp., $\left.\sigma^{\prime}\right)$ be a finitely generated subcone of $C$ (resp., $\left.C^{\prime}\right)$ such that $\sigma$ maps into $\sigma^{\prime}$ by the induced map $C \rightarrow C^{\prime}$. Assume that the natural map $\sigma \rightarrow \mathcal{S}^{\vee}$ is surjective.

Let $g: G \rightarrow G^{\prime}$ be a homomorphism of semiabelian schemes over $S$. Let $I$ (resp., $\left.I^{\prime}\right)$ be a $G$-torsor (resp., $G^{\prime}$-torsor) over $[\sigma]$ (resp., $\left.\left[\sigma^{\prime}\right]\right)$ with respect to the étale topology.

Let $f_{1}, f_{2}: I \rightarrow I^{\prime}$ be two morphisms over $S$ which are compatible with $g$ and $h$. Then, there is a constant map $c: I \rightarrow G^{\prime}$ such that $c \cdot f_{1}=f_{2}$.

Proof. Since $f_{1}$ and $f_{2}$ are compatible with $h$, there is a morphism $r: I \rightarrow$ $G^{\prime}$ such that $f_{2}(x)=r(x) f_{1}(x) \quad(x \in I)$. For $a \in G$ and $x \in I$, we have $f_{2}(a x)=g(a) f_{2}(x)=g(a) r(x) f_{1}(x)=r(x) f_{1}(a x)$. We have also $f_{2}(a x)=$ $r(a x) f_{1}(a x)$. Hence, we have $r(x)=r(a x)$, and $r$ factors through $[\sigma]$. By Lemma 9.10, the map $r:[\sigma] \rightarrow G^{\prime}$ is constant, which completes the proof. 
9.12. We now prove statement $(2)^{\prime}$ in Section 9.6.

Let $\bar{Y}$ be the image of $Y$ in $\mathcal{H o m}\left(X, \mathbb{G}_{m, \log } / \mathbb{G}_{m}\right)$, and let $\bar{X}$ be the image of $X$ in $\mathcal{H o m}\left(Y, \mathbb{G}_{m}, \log / \mathbb{G}_{m}\right)$. Then, the admissible pairing $X \times Y \rightarrow$ $\mathbb{G}_{m, \log } / \mathbb{G}_{m}$ induces a pairing $\bar{X} \times \bar{Y} \rightarrow \mathbb{G}_{m, \log } / \mathbb{G}_{m}$.

The semiabelian scheme $G$ and the homomorphism $\mathcal{S} \rightarrow \mathbb{G}_{m, \log } / \mathbb{G}_{m}$ are defined over some $S_{\lambda}$. There, the sheaves $\bar{X}, \bar{Y}$, the pairing $\bar{X} \times \bar{Y} \rightarrow$ $\mathbb{G}_{m, \log } / \mathbb{G}_{m}$, and the sheaf $\mathcal{Q}$ are defined. Let $\sigma$ be a finitely generated subcone of $C$ such that $\bigcup_{n} \ell^{n} \sigma=C$ as in Section 9.7. The $G$-torsor $I=\widetilde{A}^{(\sigma)}$ over $[\sigma]$ is also defined over some $S_{\lambda}$ because a $G$-torsor over $[\sigma]$ can be replaced with a $G$-torsor over $V(\sigma)$ with a descent data. Let the $G$-torsor $J$ over $\left[\ell^{-1} \sigma\right]$ be the restriction of $I$ to $\left[\ell^{-1} \sigma\right] \subset[\sigma]$. A map $f: J \rightarrow I$, which induces $x \mapsto x^{\ell}$ over $S$ and which satisfies the following conditions (1) and (2), is defined over some $S_{\lambda}$. We denote $f(x)$ for $x \in J$ by $x^{\ell}$, though $I$ has no multiplication.

(1) This map is compatible with $\mathcal{Q} \rightarrow \mathcal{Q} ; x \mapsto x^{\ell}$.

(2) We have $(a x)^{\ell}=a^{\ell} x^{\ell}(a \in G, x \in J)$.

Define the két sheaf of sets $\widetilde{L}$ on $S_{\lambda}$ as $\{(x, n) \mid x \in I, n \geq 0\} / \sim$, where $\sim$ is the equivalence relation generated by Proposition 9.8(1), (2). We have a surjection $\widetilde{L} \rightarrow \mathcal{Q} ;(x, n) \mapsto \operatorname{class}(x)^{\ell^{n}}$, and $\widetilde{L}$ becomes a $G$-torsor over $\mathcal{Q}$, where $a \in G$ acts by $(x, n) \mapsto(b x, n)$ with $b \in G$ such that $a=b^{\ell^{n}}$. Over $S$, we have a canonical isomorphism of $G$-torsors $\widetilde{L} \cong \widetilde{A}$ over $\mathcal{Q}$ by Proposition 9.8.

Next, a map $g: J \times J \rightarrow I$, which induces the map $(x, y) \mapsto x y$ over $S$ and satisfies the following conditions (3) and (4), is defined over some $S_{\lambda}$. We denote $g(x, y)$ for $x, y \in J$ by $x y$, though $I$ has no multiplication.

(3) This map is compatible with $\mathcal{Q} \times \mathcal{Q} \rightarrow \mathcal{Q} ;(x, y) \mapsto x y$.

(4) We have $a(x y)=(a x) y=x(a y)$ for $a \in G, x, y \in J$.

Then the following conditions are also satisfied.

(5) We have $x y=y x$ for $x, y \in J$.

(6) We have $(x y) z=x(y z)$ for $x, y, z \in K$, where $K$ is the $G$-torsor over $\left[\ell^{-2} \sigma\right]$ defined by restricting $I$ to $\left[\ell^{-2} \sigma\right] \subset[\sigma]$.

(7) We have $x^{\ell} y^{\ell}=(x y)^{\ell}$ for $x, y \in K$.

For example, to see condition (5), we apply Lemma 9.11 to $G \times G \rightarrow$ $G ;(x, y) \mapsto x y, J \times J \rightarrow I ;(x, y) \mapsto x y, J \times J \rightarrow I ;(x, y) \mapsto y x$, and so on, and prove that there is a constant $c \in G$ such that $x y=c y x$ for any $x, y$. Then, $c$ must be 1 , and we have condition (5). Conditions (6) and (7) are proved similarly. For condition (6), we apply Lemma 9.11 to $G \times G \times G \rightarrow$ 
$G ;(x, y, z) \mapsto x y z, K \times K \times K \rightrightarrows I ;(x, y, z) \mapsto(x y) z,(x, y, z) \mapsto x(y z)$, and so on. For condition (7), we apply Lemma 9.11 to $G \rightarrow G ; x \mapsto x^{\ell}, K \times K \rightrightarrows$ $I ;(x, y) \mapsto x^{\ell} y^{\ell},(x, y) \mapsto(x y)^{\ell}$, and so on.

From these properties, we see that there is a unique commutative group structure on $\widetilde{L}$ such that $(x, n)(y, n)=(x y, n)$ for any $x, y \in J$ and $n \geq 0$. Again by Lemma 9.11, it can be shown that the map $J \rightarrow I ; x \mapsto x^{\ell}$ is compatible with the $\ell$ th power map for this group structure of $\widetilde{L}$, and the natural maps $J \rightarrow \widetilde{L} ; x \mapsto(x, 0)$ and $I \rightarrow \widetilde{L} ; x \mapsto(x, 0)$. The above isomorphism $\widetilde{L} \cong \widetilde{A}$ over $S$ preserves the group structures.

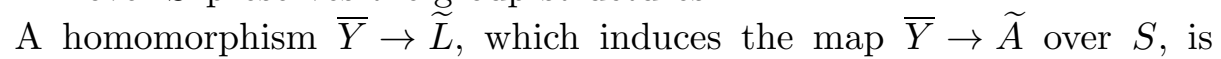
defined over some $S_{\lambda}$. Let $L=\widetilde{L} / \bar{Y}$. Over $S$, we obtain an isomorphism $L \cong A$ of group sheaves.

We show that $L$ becomes a weak $\log$ abelian variety for some $S_{\lambda}$. Definition 1.6(2) is already satisfied because there is an exact sequence $0 \rightarrow G \rightarrow$ $L \rightarrow \mathcal{Q} / \bar{Y} \rightarrow 0$.

We prove that Definition 1.6(1) for $L$ that all fibers become weak log abelian varieties holds for some $S_{\lambda}$. By dividing $S$ and $S_{\lambda}$ into the parts with locally constant $\log$ structure, we may assume that $S_{\lambda}$ has a locally constant $\log$ structure. It suffices to identify the extensions $0 \rightarrow G \rightarrow G_{\log }^{(Y)} \rightarrow \mathcal{Q} \rightarrow 0$ and $0 \rightarrow G \rightarrow \widetilde{L} \rightarrow \mathcal{Q} \rightarrow 0$. In the case of locally constant log structure, [9, Theorem 7.3(1)] implies that $\mathcal{E} x t^{1}(\mathcal{Q}, G) \cong \mathcal{H} o m(T, G)=\mathcal{H o m}(T, T)$, which is locally constant. Here $T$ is the torus part of $G$. Hence, both extensions in the above coincide in a neighborhood $U$ of the image of $S$. Replacing $\lambda$, we may assume that $U=S_{\lambda}$; that is, we may assume that they coincide.

The rest is to show that the separability condition (Definition 1.6(3)) for $L$ holds for some $S_{\lambda}$. To show this, we may assume that each $S_{\lambda}$ is of finite type over $\mathbb{Z}$. First, condition (3) is equivalent to that the zero section $S_{\lambda} \rightarrow L$ is represented by finite morphisms. Then it reduces to that the inclusion map $\bar{Y} \rightarrow \widetilde{L}$ is represented by finite morphisms. Further, it reduces to that the inclusion map $I \cap \bar{Y} \hookrightarrow I$ is represented by finite morphisms. Since each $S_{\lambda}$ is of finite type over $\mathbb{Z}$, by the part of Theorem 8.1 already proven in Section 8 (see Remark (4) after Theorem 8.1), both $I \cap \bar{Y}$ and $I=L^{(\sigma)}$ are represented by algebraic spaces with fs log structures of finite presentation over $S_{\lambda}$. Hence, the inclusion map $I \cap \bar{Y} \hookrightarrow I$ is in fact represented by finite morphisms for some $S_{\lambda}$. This completes the proof of Proposition 9.2.

9.13. We prove Theorem 8.1 in general. By Corollary 9.5, a weak log abelian variety over any base $S$ is locally a base change from a base of finite 
type over $\mathbb{Z}$. Hence, we may assume that $S$ is of finite type over $\mathbb{Z}$. This case is already proven in Section 8. Thus, the proof of Theorem 8.1 is completed.

\section{$\S 10$. Weak log abelian varieties are log algebraic spaces in some sense}

10.1. We introduce notions of log algebraic space in the first sense and in the second sense.

Let $S$ be an fs log scheme.

A log algebraic space $F$ over $S$ in the first sense is just an algebraic space with fs log structure (see Section 6.2).

We say that $F$ is $\log$ étale over $S$ if, for any fs log scheme $T$ which is strict étale over $F$, the composite $T \rightarrow F \rightarrow S$ is log étale.

A log algebraic space over $S$ in the second sense is a sheaf $F$ on (fs/ $S$ ) ét satisfying the following condition: there are a $\log$ algebraic space $F^{\prime}$ over $S$ in the first sense and a surjective morphism $F^{\prime} \rightarrow F$ of sheaves such that, for any fs $\log$ scheme $T$ and any morphism $T \rightarrow F$, the fiber product $T \times_{F} F^{\prime}$ is a log étale log algebraic space over $T$ in the first sense.

Proposition 10.2. A log algebraic space over $S$ in the first sense is a log algebraic space over $S$ in the second sense.

For the proof, see Section 6.2.

A weak log abelian variety is not just a sheaf of groups. It is a log algebraic space in the second sense as follows, though it is not necessarily a log algebraic space in the first sense.

Lemma 10.3. Let $A$ be a weak log abelian variety. Let $\tau \subset \sigma$ be two cones in $C$. Then $\widetilde{A}^{(\tau)} \rightarrow \widetilde{A}^{(\sigma)}$ is represented by log étale morphisms.

Proof. Since $V(\tau) \rightarrow V(\sigma)$ is log étale, $[\tau] \rightarrow[\sigma]$ is represented by log étale morphisms. Hence, $\widetilde{A}^{(\tau)} \rightarrow \widetilde{A}^{(\sigma)}$ is represented by log étale morphisms.

Theorem 10.4. Let $A$ be a weak log abelian variety. Then we have the following.

(1) The sheaf $A$ is a log algebraic space in the second sense.

(2) Assume that we are in the situation of Section 2.4; then, $\widetilde{A}$ is a log algebraic space in the second sense.

Proof. We may assume that we are in the situation of Sections 2.4 and 2.5.

We prove (2) first. Cover $\widetilde{A}$ by the disjoint union of the $\sigma$-parts $\widetilde{A}^{(\sigma)}$, where $\sigma$ runs over the set of all finitely generated subcones of $C$. Note 
that each $\widetilde{A}^{(\sigma)}$ is a log algebraic space in the first sense by Theorem 8.1. For two such subcones $\sigma$ and $\tau$, the fiber product $\widetilde{A}^{(\sigma)} \times{ }_{\widetilde{A}} \widetilde{A}^{(\tau)}$ is $\widetilde{A}^{(\sigma \cap \tau)}$. Since the projection $\widetilde{A}^{(\sigma \cap \tau)} \rightarrow \widetilde{A}^{(\sigma)}$ is represented by log étale morphisms by Lemma 10.3 , this proves (2).

We prove (1). Since $\bar{Y}$ is constructible, it is represented by an étale algebraic space by [5, Exposé IX, Proposition 2.7].

Let $T$ be an fs $\log$ scheme over $A$. Since $\widetilde{A}$ is étale locally isomorphic to $A \times \bar{Y}$, the fiber product $T \times_{A} \widetilde{A}$ is a $\log$ algebraic space in the first sense, which is strict étale over $T$. Since we may assume that the morphism $T \times_{A} \widetilde{A} \rightarrow \widetilde{A}$ factors through $\widetilde{A}^{(\sigma)}$ for some $\sigma$, (1) reduces to (2).

Proposition 10.5. For a log algebraic space $F$ in the second sense, for log algebraic spaces $U, V$ in the first sense, and for morphisms $U, V \rightarrow F$, the fiber product $U \times{ }_{F} V$ is a log algebraic space in the first sense.

Proof. Take a cover $F^{\prime} \rightarrow F$ from a log algebraic space in the first sense, which exists by definition. Strict étale locally on $U$, the morphism $U \rightarrow F$ factors as $U \rightarrow F^{\prime} \rightarrow F$. Thus, we may assume that $U \rightarrow F$ factors as $U \rightarrow$ $F^{\prime} \rightarrow F$. Similarly, we may assume that $V \rightarrow F$ factors as $V \rightarrow F^{\prime} \rightarrow F$. Then, $U \times{ }_{F} V$ is the fiber product of $U \times V \rightarrow F^{\prime} \times F^{\prime} \leftarrow F^{\prime} \times{ }_{F} F^{\prime}$, which is a log algebraic space in the first sense.

\subsection{Question on algebraic geometry}

A weak log abelian variety $A$ is regarded as a minimal model for the models $A^{(\Sigma)}$ for various complete fans $\Sigma$. How general can we find the minimal model in the category of log algebraic spaces in the second sense?

We will see that the moduli space of polarized log abelian variety with level structure has also such a minimal model in the category of log algebraic spaces in the second sense. This should be considered as the minimal model of various toroidal compactifications and resides over the Satake compactification. Do we have a general theory of nice minimal compactification of an algebraic variety in the category of log algebraic spaces in the second sense?

\section{§11. Valuative criterion for weak log abelian varieties}

In this section, we give a sufficient condition for a model of a weak log abelian variety to be proper.

11.1. We recall the definition of the direct image log structure. Let $f: T \rightarrow S$ be a morphism of schemes, and let $N$ be a $\log$ structure on $T$. 
Let $f_{*} N$ be the direct image sheaf of $N$. Then we define the direct image $\log$ structure of $N$ as the fiber product $M$ of $f_{*} N \rightarrow f_{*} \mathcal{O}_{T} \leftarrow \mathcal{O}_{S}$.

11.2. Let $K$ be a complete discrete valuation field with valuation ring $O_{K}$

Fix an fs $\log$ structure $N$ on $\eta:=\operatorname{Spec}(K)$, and let $M$ be the log structure on $\operatorname{Spec}\left(O_{K}\right)$ defined to be the direct image of $N$. If $N$ is trivial, $M$ is the standard $\log$ structure of $\operatorname{Spec}\left(O_{K}\right)$. If $N$ is not trivial, then $M$ is not an fs $\log$ structure. If we denote by $\mathcal{S}$ the stalk of $N / \mathcal{O}_{\eta}^{\times}$, which is a sharp fs monoid, and if we denote the monoid law of $\mathcal{S}$ additively, then the stalk of $M / \mathcal{O}_{\mathrm{Spec}\left(O_{K}\right)}^{\times}$over the closed point of $\operatorname{Spec}\left(O_{K}\right)$ is isomorphic to the monoid $\{(x, y) \in \mathcal{S} \times \mathbb{Z} \mid y \geq 0$ if $x=0\}$. Here $(0,1) \in \mathcal{S} \times \mathbb{Z}$ corresponds to the class of a prime element of $K$, and the canonical projection to the stalk of $N / \mathcal{O}_{\eta}^{\times}$ corresponds to the projection $\mathcal{S} \times \mathbb{Z} \rightarrow \mathcal{S}$. For example, if $\mathcal{S}=\mathbb{N}$, this stalk of $M / \mathcal{O}_{\operatorname{Spec}\left(O_{K}\right)}^{\times}$is isomorphic to the monoid of all positive elements of $\mathbb{Z}^{2}$ for the lexicographic order, and it is not a finitely generated monoid.

From now on, assume that $N$ is charted by $\mathcal{S}$. Then, $M$ is the filtered union of fs $\log$ structures contained in $M$. Further, $M$ is the filtered union of fs $\log$ structures contained in $M$ whose restriction on $\eta$ is $N$. Let $A$ be a weak log abelian variety over an fs log scheme $S$. For a morphism $\left(\operatorname{Spec}\left(O_{K}\right), M\right) \rightarrow S$ of $\log$ schemes, let $A\left(O_{K}\right):=\lim _{\longrightarrow} A\left(\left(\operatorname{Spec}\left(O_{K}\right), M_{i}\right)\right)$, where $M_{i}$ runs over the set of fs log structures contained in $M$ whose restriction on $\eta$ is $N$.

In this section, we prove the following.

Proposition 11.3. Let $A$ be a weak log abelian variety over an $f s$ log scheme $S$. Assume that we are in the situation of Sections 2.4 and 2.5. Let $\Sigma$ be a complete fan. Assume that $A$ satisfies the following condition.

(*) Let $K$ be a complete discrete valuation field. Let $N$ be an $f s$ log structure on $\eta=\operatorname{Spec}(K)$. Suppose that $N$ is charted by the stalk of $N / \mathcal{O}_{\eta}^{\times}$. Let $M$ be the direct image log structure of $N$ on $\operatorname{Spec}\left(O_{K}\right)$. Then, $A\left(O_{K}\right)=A(K)$ for any morphism $\left(\operatorname{Spec}\left(O_{K}\right), M\right) \rightarrow S$ of log schemes.

Then the algebraic space $A^{(\Sigma)}$ with an fs log structure is proper and log smooth over $S$.

Remark. We will prove in Theorem 17.1 that $(*)$ is always satisfied.

In the rest of this section, we prove Proposition 11.3. The log smoothness is satisfied (see Remark (3) after Theorem 8.1). We prove the properness by checking the valuative criterion as follows. 
First, we may assume that $S$ is locally Noetherian because a weak log abelian variety over any base is locally a base change from a base of finite type over $\mathbb{Z}$ (see Corollary 9.5).

We show that $A^{(\Sigma)}$ is separated over $S$ by using the separatedness of $A$. By definition, the diagonal morphism $A \rightarrow A \times{ }_{S} A$ is represented by finite morphisms. Since the diagonal morphism $A^{(\Sigma)} \rightarrow A^{(\Sigma)} \times{ }_{S} A^{(\Sigma)}$ for $A^{(\Sigma)}$ is the base change of that for $A$ (because $A^{(\Sigma)}$ is a subsheaf of $A$ ), it is also represented by finite morphisms. Since $\left(A^{(\Sigma)} \times{ }_{S} A^{(\Sigma)}\right)^{\circ} \rightarrow\left(A^{(\Sigma)}\right)^{\circ} \times_{\circ_{S}}\left(A^{(\Sigma)}\right)^{\circ}$ is finite, the diagonal morphism for $\left(A^{(\Sigma)}\right)^{\circ} \rightarrow\left(A^{(\Sigma)}\right)^{\circ} \times{ }_{S}\left(A^{(\Sigma)}\right)^{\circ}$ of algebraic spaces is also finite, hence a closed immersion; that is, $\left(A^{(\Sigma)}\right)^{\circ}$ is separated.

To see that $\left(A^{(\Sigma)}\right)^{\circ}$ is universally closed over $\stackrel{\circ}{S}$, we recall the valuative criterion for algebraic spaces.

Proposition 11.4. Let $Y$ be a locally Noetherian scheme. Let $f: X \rightarrow Y$ be a separated morphism of algebraic spaces. Assume that

(1) $f$ is of finite type, and

(2) for any commutative diagram

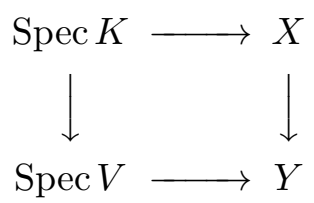

where $V$ is a discrete valuation ring with the field of fractions $K$, there exist a finite separable extension $K \subset K^{\prime}$ of fields, a discrete valuation ring $V^{\prime} \subset K^{\prime}$ dominating $V$, and a morphism Spec $V^{\prime} \rightarrow X$ such that $\operatorname{Spec} K^{\prime} \rightarrow$ Spec $K \rightarrow X$ coincides with $\operatorname{Spec} K^{\prime} \rightarrow \operatorname{Spec} V^{\prime} \rightarrow X$ and Spec $V^{\prime} \rightarrow X \rightarrow Y$ coincides with $\operatorname{Spec} V^{\prime} \rightarrow \operatorname{Spec} V \rightarrow Y$.

Then $f$ is universally closed and, hence, proper.

The proof is parallel to the scheme case [6, Théorème 7.3.8] based on the Chow's lemma for algebraic spaces [15, Chapter IV, Theorem 3.1] (see [6, Corollaire 5.6.3] and [3, Lemma 41.1]).

11.5. By Proposition 11.4, it is enough to check Proposition 11.4(1)-(2) for $A^{(\Sigma)} \rightarrow S$.

First we prove Proposition 11.4(1). We already know that $A^{(\Sigma)}$ is locally of finite type (see Section 8.4). To prove that $A^{(\Sigma)} \rightarrow S$ is quasicompact, we may assume that $S$ is affine and has a chart, and it is enough to show that $A^{(\Sigma)}$ is quasicompact. In this case, each constant $\log \operatorname{locus}$ of $S$ is 
quasicompact. Hence, we may further assume that the $\log$ of $S$ is constant. By condition (3) in Section 3.1, there are a finite number of cones $\Delta_{1}, \ldots, \Delta_{n}$ such that $\Sigma$ consists of all the $Y$-translations of all their faces. Then, the proof of Theorem 4.1 shows that $\bigcup_{i} \widetilde{A}^{\left(\Delta_{i}\right)} \rightarrow A^{(\Sigma)}$ is surjective and that $A^{(\Sigma)}$ is represented by a quasicompact fs log scheme.

Next, we prove that $A^{(\Sigma)} \rightarrow S$ satisfies Proposition 11.4(2). It is sufficient to prove the following.

11.6. Assume that the underlying scheme of the base $S$ is $\operatorname{Spec}(V)$, where $V$ is a complete discrete valuation ring with separably closed residue field. Let $K$ be the field of fractions of $V$, and assume that we have a morphism $a$ from $\operatorname{Spec}(K)$ to the underlying algebraic space $\left(A^{(\Sigma)}\right)^{\circ}$ of $A^{(\Sigma)}$ over the underlying scheme $S^{\circ}$ of $S$. Assume that there is a chart $P \rightarrow N$ such that $P \rightarrow N(K) / K^{\times}$is bijective, where $N$ is the inverse image log structure on $\operatorname{Spec}(K)$ from $A^{(\Sigma)}$ via $a$. Then, there is a morphism $b: S^{\circ} \rightarrow\left(A^{(\Sigma)}\right)^{\circ}$ over $S^{\circ}$ which is compatible with $a$.

11.7. We prove the statement of Section 11.6. Endow $\operatorname{Spec}(K)$ with the pullback $\log$ structure $N$ from $A^{(\Sigma)}$ via $a$. Then, we have a section $a^{\prime} \in$ $A^{(\Sigma)}(K)$ whose underlying morphism is $a$. Let $M_{i}$ be as in Section 11.2. Let $S_{i}:=\left(\operatorname{Spec}\left(O_{K}\right), M_{i}\right)$. By definition and the assumption, $\lim _{\longrightarrow} A\left(S_{i}\right)=$ $A\left(O_{K}\right)=A(K)$. Hence, for some $i$, we have an element $a^{\prime \prime}$ of $A\left(S_{i}\right)$ whose image in $A(K)$ coincides with that of $a^{\prime}$. By Proposition 3.2, there are a log blowing up $S^{\prime} \rightarrow S_{i}$ and an element of $A^{(\Sigma)}\left(S^{\prime}\right)$ whose image in $A\left(S^{\prime}\right)$ coincides with that of $a^{\prime \prime}$. Take a $V$-rational point $S^{\circ} \rightarrow\left(S^{\prime}\right)^{\circ}$ of $\left(S^{\prime}\right)^{\circ}$. Denote by $S^{\prime \prime}$ the $S^{\circ}$ endowed with the pullback log structure from $S^{\prime}$. Let $\eta^{\prime \prime}$ be its generic point, that is, $\operatorname{Spec}(K)$ endowed with the pullback $\log$ structure from $S^{\prime}$. Then, by the injectivity of $A^{(\Sigma)}\left(S^{\prime \prime}\right) \rightarrow A^{(\Sigma)}\left(\eta^{\prime \prime}\right)$, the restriction of $a^{\prime}$ to $A^{(\Sigma)}\left(\eta^{\prime \prime}\right)$ lifts to $A^{(\Sigma)}\left(S^{\prime \prime}\right)$. This proves the statement of Section 11.6 and completes the proof of Proposition 11.3.

\section{§12. Proper models with constant degeneration}

Here we prove that a complete fan yields a proper model in the case of constant degeneration. The general case will be proved in Theorem 17.1.

Proposition 12.1. Let $A$ be a weak log abelian variety with constant degeneration over an $f$ s log scheme $S$. Assume that we are in the situation of Sections 2.4 and 2.5. Let $\Sigma$ be a complete fan. Then the log smooth algebraic space $A^{(\Sigma)}$ with fs log structure is proper over $S$. 
We prove the converse in Proposition 12.7.

Since we can take a complete fan $\Sigma$ by [8, Theorem 5.2.1], we have the following.

Corollary 12.2. Let $A$ be a weak log abelian variety with constant degeneration. Then a proper model of $A$ exists.

We remark that, if $M_{S} / \mathcal{O}_{S}^{\times}$is locally constant, then there is a proper model which is a scheme (see Corollary 4.2).

12.3. We prove Proposition 12.1. By Proposition 11.3, it is enough to check that $A\left(O_{K}\right)=A(K)$. Since $A$ is with constant degeneration, by definition it corresponds to a log 1-motif $\left[Y \rightarrow G_{\log }\right]$, which induces an admissible and nondegenerate pairing $X \times Y \rightarrow \mathbb{G}_{m, \log }$. Here $X$ and $Y$ are locally free sheaves of $\mathbb{Z}$-modules on $S$.

First we reduce to

$$
T_{\log }^{(Y)}\left(O_{K}\right)=T_{\log }^{(Y)}(K),
$$

where the left-hand side is defined by the limit similarly as in Section 11.2. Assume that $(*)$ is valid. Since we have the exact sequence $0 \rightarrow T_{\log }^{(Y)} \rightarrow$ $G_{\log }^{(Y)} \rightarrow B \rightarrow 0$, and $B\left(O_{K}\right)=B(K),(*)$ implies that $G_{\log }^{(Y)}\left(O_{K}\right)=G_{\log }^{(Y)}(K)$. Then, by dividing $Y$, we have $A\left(O_{K}\right)=A(K)$. (Here we use $A=G_{\log }^{(Y)} / Y$ and $H_{\text {ét }}^{1}(\operatorname{Spec} K, Y)=H_{\text {ét }}^{1}\left(\operatorname{Spec} \mathcal{O}_{K}, Y\right)=0$.)

To show $(*)$, by replacing $K$ with an unramified extension, we may and will assume that $X$ and $Y$ are free. We first prove that

$$
T_{\log }\left(O_{K}\right) \stackrel{\sim}{\rightarrow} T_{\log }(K) .
$$

To this end, we may assume that $X=\mathbb{Z}$, and (**) reduces to $M^{\mathrm{gp}}\left(O_{K}\right)=$ $N^{\mathrm{gp}}(K)$ in the notation of Section 11.2. We fix a chart of $N$ by $\mathcal{S}$ as in Section 11.2, and we also fix a prime element of $K$. Then, the map $M^{\mathrm{gp}}\left(O_{K}\right) \rightarrow N^{\mathrm{gp}}(K)$ is naturally decomposed into $M^{\mathrm{gp}}\left(O_{K}\right)=\mathcal{S}^{\mathrm{gp}} \times \mathbb{Z} \times$ $O_{K}^{\times}=\mathcal{S}^{\mathrm{gp}} \times K^{\times}=N^{\mathrm{gp}}(K)$. Hence, $(* *)$ holds.

Therefore, to see $(*)$, it suffices to show that, for a map $\varphi: X \rightarrow \mathbb{G}_{m, \log }$ on $O_{K}$, if its restriction on $K$ belongs to the $(Y)$-part, it itself belongs to the $(Y)$-part on $O_{K}$. Thus, the problem is reduced to the following lemma by fixing a prime element of $K$ and identifying $K^{\times}$with $\mathbb{Z} \times O_{K}^{\times}$.

LEMMA 12.4. Let $K$ be a complete discrete valuation field. Let $\mathcal{S}$ be a sharp fs monoid. Let $V$ be an fs submonoid of $M:=\{(x, y) \in \mathcal{S} \times \mathbb{Z} \mid y \geq$ 
0 if $x=1\}$ such that $\{1\} \times \mathbb{N} \subset V$ and $V^{\mathrm{gp}}=\mathcal{S}^{\mathrm{gp}} \times \mathbb{Z}$. Let $X$ and $Y$ be free $\mathbb{Z}$-modules of finite rank, and let

$$
\langle,\rangle: X \times Y \rightarrow \mathcal{S}^{\mathrm{gp}} \times \mathbb{Z}
$$

be an admissible and nondegenerate pairing with respect to $V$. Let $\varphi: X \rightarrow$ $\mathcal{S}^{\mathrm{gp}} \times \mathbb{Z}$ be a homomorphism. Assume that, for any $x \in X$, there are $y_{1}, y_{2} \in$ $Y$ such that $\left.\left.\left\langle x, y_{1}\right\rangle\right|_{K} \varphi(x)\right|_{K}\left\langle x, y_{2}\right\rangle$, where $\left.a\right|_{K} b$ means that $b / a$ belongs to $\mathcal{S} \times \mathbb{Z}$. Then, for any $x \in X$, there are $y_{1}, y_{2} \in Y$ such that $\left\langle x, y_{1}\right\rangle|\varphi(x)|$ $\left\langle x, y_{2}\right\rangle$, where $a \mid b$ means that $b / a$ belongs to $M$.

Proof. Throughout the proof, we fix an $x \in X$. Let $h$ be the projection $V \rightarrow \mathcal{S} ;(s, n) \mapsto s$. If $h(\langle x, Y\rangle) \cap \mathcal{S} \neq\{1\}$, then there is an element $y \in Y$ with $\langle x, y\rangle \in(\mathcal{S} \backslash\{1\}) \times \mathbb{Z}$. Then we have $\left\langle x, y_{1} y^{-1}\right\rangle|\varphi(x)|\left\langle x, y_{2} y\right\rangle$. Indeed,

$$
\begin{gathered}
\varphi(x)\left\langle x, y_{1} y^{-1}\right\rangle^{-1}=\varphi(x)\left\langle x, y_{1}^{-1}\right\rangle\langle x, y\rangle \in(S \backslash\{1\}) \times \mathbb{Z} \quad \text { and } \\
\varphi(x)^{-1}\left\langle x, y_{2} y\right\rangle=\varphi(x)^{-1}\left\langle x, y_{2}\right\rangle\langle x, y\rangle \in(S \backslash\{1\}) \times \mathbb{Z} .
\end{gathered}
$$

If $h(\langle x, Y\rangle) \cap \mathcal{S}=\{1\}$, then we take a homomorphism $N: \mathcal{S} \rightarrow \mathbb{R}_{\geq 0}$ such that $\operatorname{Ker} N^{\mathrm{gp}} \supset h(\langle x, Y\rangle)$ and $\operatorname{Ker} N^{\mathrm{gp}} \cap \mathcal{S}=\{1\}$. Let $\sigma=\operatorname{Ker}(N h)$. By the admissibility, the pairing

$$
X / X_{\sigma} \times Y / Y_{\sigma} \rightarrow \mathbb{R}
$$

induced by $N h$ is nondegenerate. Hence, $x \in X_{\sigma}$. Note that $\sigma=\{1\} \times \mathbb{N} \subset V$. Again by the admissibility, we have the nondegenerate pairing $X_{\sigma} \times Y_{\sigma} \rightarrow \mathbb{R}$ induced by a nontrivial homomorphism $\sigma \rightarrow \mathbb{R}_{\geq 0}$. So there exists $y \in Y$ with $\langle x, y\rangle=(1, m)$ for a sufficiently large $m \in \mathbb{N}$. Hence, we conclude that $\left\langle x, y_{1} y^{-1}\right\rangle|\varphi(x)|\left\langle x, y_{2} y\right\rangle$ in the same way as above.

This completes the proof of Proposition 12.1. To prove the converse in Proposition 12.7 below, we first show lemmas.

Lemma 12.5. Let the situation be as in Sections 2.4 and 2.5. Let $\Sigma$ be a fan in $C$, and let $|\Sigma|$ be the $\mathbb{R}_{\geq 0}$-support of $\Sigma$. Let $s \in S$. The homomorphism $\mathcal{S} \rightarrow M_{S, \bar{s}} / \mathcal{O}_{S, \bar{s}}^{\times}$induces $\operatorname{Hom}\left(M_{S, \bar{s}} / \mathcal{O}_{S, \bar{s}}^{\times}, \mathbb{N}\right) \rightarrow \operatorname{Hom}(\mathcal{S}, \mathbb{N})$. Let $N$ be an element of the interior of $\operatorname{Hom}\left(M_{S, \bar{s}} / \mathcal{O}_{S, \bar{s}}^{\times}, \mathbb{N}\right)$, and let $|\Sigma|_{N}$ be the set of all elements of $|\Sigma|$ whose image in $\operatorname{Hom}\left(\mathcal{S}, \mathbb{R}_{\geq 0}\right)$ coincides with the image of $N$.

Then there is a natural bijection between the following two sets: 
(1) the set of connected components of the fiber of $A^{(\Sigma)}$ (resp., $\left.\widetilde{A}^{(\Sigma)}\right)$ over $s$; and

(2) the set of connected components of $\bar{Y}_{\bar{s}} \backslash|\Sigma|_{N}$ (resp., $|\Sigma|_{N}$ ).

Proof. We describe the case of $A^{(\Sigma)}$. The proof for $\widetilde{A}^{(\Sigma)}$ is similar.

There is a natural bijection between set (2) and the following set (3). Endow $\Sigma$ and the set $\operatorname{Spec}(\mathcal{S})$ of faces of $\mathcal{S}$ with Zariski topologies.

(3) The set of connected components of the fiber of $\Sigma \rightarrow \operatorname{Spec}(\mathcal{S})$ over the point of $\operatorname{Spec}(\mathcal{S})$ which is the image of the unique closed point of $\operatorname{Spec}\left(M_{S, \bar{s}} / \mathcal{O}_{S, \bar{s}}^{\times}\right)$.

By the exact sequence $0 \rightarrow G \rightarrow A^{(\Sigma)} \rightarrow \bar{Y} \backslash \mathcal{Q}^{(\Sigma)} \rightarrow 0$, since all fibers of $G$ are connected, we have a natural bijection between set (1) and set (3).

LEMMA 12.6. Let $\Sigma$ be a complete fan which is stable under the action of $Y$. Then all fibers of $A^{(\Sigma)}$ (resp., $\widetilde{A}^{(\Sigma)}$ ) are connected.

Proof. In this case, $|\Sigma|=C \otimes \mathbb{R}_{\geq 0}$, and hence $|\Sigma|_{N}$ is connected for any $N$. Hence, this lemma follows from Lemma 12.5.

We prove the converse of Proposition 12.1.

Proposition 12.7. Let $A$ be a weak log abelian variety with constant degeneration over a nonempty fs log scheme $S$. Assume that we are in the situation of Sections 2.4 and 2.5. Let $\Sigma$ be a Y-stable fan. Then $\Sigma$ is complete if the log smooth algebraic space $A^{(\Sigma)}$ with $f_{s} \log$ structure is proper over $S$.

Proof. Assume that $A^{(\Sigma)}$ is proper over $S$. Take a complete fan $\Sigma^{\prime}$ by $[8$, Theorem 5.2.1]. By [8, Lemma 5.2.14], we may assume that $\Sigma^{\prime}$ contains a subdivision $\Sigma^{\prime \prime}$ of $\Sigma$ as a subfan. Consider the diagram

$$
A^{(\Sigma)} \stackrel{p}{\leftarrow} A^{\left(\Sigma^{\prime \prime}\right)} \stackrel{i}{\rightarrow} A^{\left(\Sigma^{\prime}\right)} .
$$

By Proposition 8.8, we know that $p$ is proper and that $i$ is a strict open immersion. Since both $A^{\left(\Sigma^{\prime \prime}\right)}$ and $A^{\left(\Sigma^{\prime}\right)}$ are proper by the assumption and Proposition 12.1, respectively, Lemma 12.6 implies that $A^{\left(\Sigma^{\prime \prime}\right)}$ and $A^{\left(\Sigma^{\prime}\right)}$ coincide so that $\Sigma^{\prime \prime}=\Sigma^{\prime}$, this implies that $\Sigma$ is complete.

\section{$\S 13$. Weak log abelian varieties over complete discrete valuation rings, I}

To prove the existence of a proper model for a general weak log abelian variety with nonconstant degeneration, we study weak log abelian varieties 
over complete discrete valuation rings. First we state our results, some of which will be proved in a future part of this series.

13.1. Assume that we are in the situation of Section 11.2. In particular, Spec $O_{K}$ is endowed with the direct image $\log$ structure $M$. Let $M_{i}$ be as in Section 11.2.

Let $\mathcal{C}_{0}$ be the category of weak log abelian varieties over $O_{K}$, which is defined to be the inductive limit of the categories of weak log abelian variety for fs log structures $M_{i}$. The polarizability can be understood as "polarizable for some $M_{i}$."

Let $\mathcal{C}_{1}$ be the category of admissible $\log 1$-motifs over $O_{K}$ with respect to the $\log$ structure $M$, which is defined similarly.

Let $(\mathrm{WLAV} / K$ ) be the category of weak log abelian varieties over $K$ with respect to the log structure $N$.

Let $\mathcal{C}_{2}$ be the full subcategory of $(\mathrm{WLAV} / K$ ) consisting of objects having the following property: if $\left[Y \rightarrow G_{\log }\right]$ denotes the corresponding log 1-motif over $K$ and $0 \rightarrow T \rightarrow G \rightarrow B \rightarrow 0$ denotes the exact sequence with $T$ a torus and $B$ an abelian variety, then $Y$ and $T$ are unramified and $B$ is of semistable reduction.

13.2. We have the following functors $\alpha_{i}: \mathcal{C}_{0} \rightarrow \mathcal{C}_{i}(i=1,2)$.

The $\alpha_{2}$ is the evident functor.

The definition of $\alpha_{1}$ is as follows. If we are given a weak log abelian variety over $O_{K}$ with respect to $M$, then for each $n \geq 1$, we have a weak log abelian variety over $O_{K} / m_{K}^{n}$, and the corresponding admissible log 1motif over $O_{K} / m_{K}^{n}$, where $m_{K}$ is the maximal ideal of $O_{K}$. This family of admissible log 1-motifs for $n \geq 1$ defines an admissible log 1-motif over $O_{K}$.

13.3. In the present article, we prove that there is a category equivalence $\mathcal{C}_{1}^{[\mathrm{pol}]} \simeq \mathcal{C}_{2}$ compatible with $\alpha_{1}$ and $\alpha_{2}$, where $\mathcal{C}_{1}^{[\text {pol }]}$ is the following subcategory of $\mathcal{C}_{1}$.

Let $\left[Y_{1} \rightarrow G_{\log }\right]$ be an object of $\mathcal{C}_{1}$.

Let $Y_{(1)}$ be the kernel of the canonical homomorphism $Y_{1} \rightarrow Y_{2}$, where $Y_{2}$ is the stalk of $\bar{Y}_{1}$ at the generic point.

Let $T_{2} \subset T$ be the torus corresponding to the stalk $X_{2}$ of $\bar{X}_{1}$ at the generic point, where $T$ is the torus part of $G$.

Let $G_{(1)}=G / T_{2}$.

The composition $Y_{(1)} \rightarrow Y_{1} \rightarrow G_{\log } \rightarrow G_{(1), \log }$ is an admissible log 1-motif. 
Then, $\left[Y_{1} \rightarrow G_{\text {log }}\right]$ belongs to $\mathcal{C}_{1}^{[\mathrm{pol}]}$ if and only if this $\log 1$-motif $Y_{(1)} \rightarrow$ $G_{(1), \log }$ is polarizable. (Here [pol] means the partial polarization on the part corresponding to the abelian part $B_{2}$ of the corresponding object in $\mathcal{C}_{2}$.)

13.4. In a future article of this series, we will prove more details of the following.

For $i=0,1,2$, let $\mathcal{C}_{i}^{\text {ptpol }}$ be the full subcategory of $\mathcal{C}_{i}$ consisting of pointwise polarizable objects, and let $\mathcal{C}_{i}^{\text {pol }}$ be the full subcategory of $\mathcal{C}_{i}$ consisting of polarizable objects. In particular, $C_{0}^{\text {ptpol }}$ is the category of $\log$ abelian varieties over $O_{K}$. Evidently, for $i=0,1,2$, we have $\mathcal{C}_{i} \supset \mathcal{C}_{i}^{\text {ptpol }} \supset \mathcal{C}_{i}^{\text {pol }}$, and, for $i=1,2$, the functor $\alpha_{i}$ sends $\mathcal{C}_{0}^{\text {pol }}$ (resp., $\mathcal{C}_{0}^{\text {ptpol }}$ ) into $\mathcal{C}_{i}^{\text {pol }}\left(\right.$ resp., $\left.\mathcal{C}_{i}^{\text {ptpol }}\right)$.

In a future serial, we will prove that $\mathcal{C}_{0}^{\text {pol }}=\mathcal{C}_{0}^{\text {ptpol }}$ and that, for $i=1,2$, $\alpha_{i}$ induces an equivalence of categories $\mathcal{C}_{0}^{\text {pol }} \simeq \mathcal{C}_{i}^{\text {pol }}$.

Summing up these results, we will have

$$
\begin{aligned}
& \mathcal{C}_{1} \supset \mathcal{C}_{1}^{[\mathrm{pol}]} \supset \mathcal{C}_{1}^{\text {ptpol }} \supset \mathcal{C}_{1}^{\text {pol }} \\
& \alpha_{1} \uparrow \quad \uparrow \quad \cong \uparrow(*) \\
& \mathcal{C}_{0} \supset \mathcal{C}_{0}^{\text {ptpol }} \stackrel{(*)}{=} \mathcal{C}_{0}^{\text {pol }} \\
& \alpha_{2} \downarrow \quad \cong \downarrow(*) \quad \cong \downarrow(*) \\
& \mathcal{C}_{2} \supset \mathcal{C}_{2}^{\text {ptpol }}=\mathcal{C}_{2}^{\text {pol }} \text { and } \mathcal{C}_{1}^{[\text {pol }]} \simeq \mathcal{C}_{2} \text {, }
\end{aligned}
$$

where the equivalences with $(*)$ will be proved in a forthcoming part of this series.

In the case where $N$ is trivial, $\mathcal{C}_{2}=\mathcal{C}_{2}^{\text {ptpol }}$. Hence, by taking the generic fiber, we have an equivalence from the category of log abelian varieties over $O_{K}$ for the standard log structure to the category of abelian varieties over $K$ with semistable reduction.

\section{\$14. Weak log abelian varieties over complete discrete valuation rings, II}

We will prove that $\mathcal{C}_{1}^{[\text {pol] }} \simeq \mathcal{C}_{2}$ in Theorem 15.10 in the next section.

14.1. First, we prove the equivalence of classical parts $\mathcal{C}_{1}^{0,[\mathrm{pol}]} \simeq \mathcal{C}_{2}^{0}$ of both categories by using the Raynaud extension, where the classical parts are defined as follows.

Let the notation be as in Section 13.

Let $\mathcal{C}_{2}^{0}$ be the full subcategory of $\mathcal{C}_{2}$ consisting of abelian varieties over $K$ of semistable reduction. 
Let $\mathcal{C}_{1}^{0,[\mathrm{pol}]}$ be the full subcategory of $\mathcal{C}_{1}^{[\mathrm{pol}]}$ consisting of objects $\left[Y \rightarrow G_{\log }\right]$ such that the image of $Y$ is contained in $G(K) \subset G_{\log }\left(O_{K}\right)$ after a finite unramified extension of $K$.

Thus, $\mathcal{C}_{1}^{0,[\mathrm{pol}]}$ (resp., $\mathcal{C}_{2}^{0}$ ) coincides with the $\mathcal{C}_{1}^{[\mathrm{pol}]}$ (resp., $\mathcal{C}_{2}$ ) of the case where $N$ is the trivial $\log$ structure on $\operatorname{Spec}(K)$.

14.2. We have the classical equivalence $\mathcal{C}_{1}^{0,[\mathrm{pol}]} \simeq \mathcal{C}_{2}^{0}$. If $\left[Y \rightarrow G_{\log }\right]$ is an object of $\mathcal{C}_{1}^{0,[\mathrm{pol}]}$ with constant $Y$, the corresponding object $A$ in $\mathcal{C}_{2}^{0}$ satisfies $A(K)=Y \backslash G(K)$ and $A^{\text {an }}=Y \backslash G_{K}^{\text {an }}$ in the category of rigid analytic groups. Here and hereafter, the "an" represents the rigid analytification.

14.3. For this equivalence, we could refer to the result " $D D=D E G "$ in [4, Chapter III]. Here we see the equivalence by Raynaud's theory. By [20, Théorème 1], there is a functor (Raynaud's construction)

$$
\delta: \mathcal{C}_{2}^{0} \rightarrow \mathcal{C}_{1}^{0,[\mathrm{pol}]}
$$

Let $\mathcal{C}_{3}^{0}$ be the category of rigid analytic abelian varieties over $K$. Consider the natural functors $\beta_{1}: \mathcal{C}_{1}^{0,[\mathrm{pol}]} \rightarrow \mathcal{C}_{3}^{0}$, which is fully faithful, and $\beta_{2}: \mathcal{C}_{2}^{0} \rightarrow$ $\mathcal{C}_{3}^{0}$, which is easily seen to be an equivalence. Since the composite $\beta_{1} \delta$ coincides with $\beta_{2}$, the functor $\delta$ is an equivalence.

\section{$\S 15$. Weak log abelian varieties over complete discrete valuation rings, III}

We prove that $\mathcal{C}_{1}^{[\mathrm{pol}]} \simeq \mathcal{C}_{2}$. Let the notation be as in Section 13 .

First, we construct a functor $\gamma: \mathcal{C}_{1}^{[\mathrm{pol}]} \rightarrow \mathcal{C}_{2}$.

15.1. Let $\left[Y_{1} \rightarrow G_{1, \log }\right]$ be an object of $\mathcal{C}_{1}^{[\mathrm{pol}]}$ with an exact sequence $0 \rightarrow T_{1} \rightarrow G_{1} \rightarrow B_{1} \rightarrow 0$ with $T_{1}$ a torus and $B_{1}$ an abelian scheme over $O_{K}$. We define the log 1 -motif $\left[Y_{2} \rightarrow G_{2, \log }\right]$ over $K$ corresponding to the associated object of $\mathcal{C}_{2}$.

15.2. Let $\left[X_{1} \rightarrow G_{1, \log }^{*}\right]$ be the dual $\log 1$-motif of $\left[Y_{1} \rightarrow G_{1, \log }\right]$ with an exact sequence $0 \rightarrow T_{1}^{*} \rightarrow G_{1}^{*} \rightarrow B_{1}^{*} \rightarrow 0$, where $T_{1}^{*}$ is a torus and $B_{1}^{*}$ is an abelian scheme over $O_{K}$. So, $X_{1}$ is the character group of $T_{1}, Y_{1}$ is the character group of $T_{1}^{*}$, and $B_{1}^{*}$ is the dual abelian scheme of $B_{1}$.

Let $Y_{(1)} \subset Y_{1}$ be the kernel of the restriction of $Y_{1} \rightarrow G_{1, \log } / G_{1}$ to $\operatorname{Spec}(K)$, and define $X_{(1)} \subset X_{1}$ similarly.

Let $Y_{2}=Y_{1} / Y_{(1)}$, and let $X_{2}=X_{1} / X_{(1)}$. Let $T_{2}$ be the torus whose character group is $X_{2}$, and let $T_{2}^{*}$ be the torus whose character group is $Y_{2}$. Thus, 
$T_{2} \subset T_{1}$ and $T_{2}^{*} \subset T_{1}^{*}$. Define semiabelian schemes $G_{(1)}$ and $G_{(1)}^{*}$ over $O_{K}$ as $G_{(1)}=G_{1} / T_{2}$ and $G_{(1)}^{*}=G_{1}^{*} / T_{2}^{*}$, respectively. Then $\left[Y_{(1)} \rightarrow G_{(1), \log }\right]$ and $\left[X_{(1)} \rightarrow G_{(1), \log }^{*}\right]$ are objects of $\mathcal{C}_{1}^{0,[\mathrm{pol}]}$. Let $B_{2}$ and $B_{2}^{*}$ be the corresponding objects of $\mathcal{C}_{2}^{0}$ by the classical equivalence in Section 14 .

The image of the composition $X_{1} \rightarrow G_{1, \log }^{*} \rightarrow G_{(1), \log }^{*}$ is contained in $G_{(1)}^{*}(K)$ after a finite unramified extension of $K$, and hence, we have a homomorphism $X_{2}=X_{1} / X_{(1)} \rightarrow\left(X_{(1)} \backslash G_{(1)}^{*}\right)(K)=B_{2}^{*}(K)$. Let $G_{2}$ be the extension of $B_{2}$ by $T_{2}$ over $K$ corresponding to this homomorphism $X_{2} \rightarrow B_{2}^{*}$.

Remark. The object $\left[Y_{(1)} \rightarrow G_{(1), \log }\right]$ already appeared in Section 13.3. An alternative construction of the extension $0 \rightarrow T_{2} \rightarrow G_{2} \rightarrow B_{2} \rightarrow 0$ is as follows. Take a proper model $P$ of $B_{2}$ associated to a star (see [4, Chapter III, Section 3]). Then, the formal $T_{2}$-torsor on $P$ given by $\left[Y_{1} \rightarrow G_{1, \log }\right]$ is algebraizable, and hence, we have a $T_{2}$-torsor on $P$. Thus, we have the desired extension.

15.3. We define a homomorphism $Y_{2} \rightarrow G_{2, \log }$. Note that $G_{2, \log }$ represents the functor of the pairs $(E, s)$, where $E$ is an extension of $B_{2}^{*}$ by $\mathbb{G}_{m}$ and $s$ is a homomorphism $X_{2} \rightarrow E_{\log }$ (here $E_{\log }$ denotes the pushout of $\mathbb{G}_{m, \log } \leftarrow \mathbb{G}_{m} \rightarrow E$ ) which lifts $X_{2} \rightarrow B_{2}^{*}$. Let $a \in Y_{2}$. Then $a$ defines a homomorphism $T_{2}^{*} \rightarrow \mathbb{G}_{m}$, and hence, from the exact sequence $0 \rightarrow T_{2}^{*} \rightarrow$ $G_{2}^{*} \rightarrow B_{2}^{*} \rightarrow 0$, we obtain an extension $E$ of $B_{2}^{*}$ by $\mathbb{G}_{m}$. The homomorphism $X_{2} \rightarrow G_{2, \log }^{*}$ induces $X_{2} \rightarrow E_{\log }$ which lifts $X_{2} \rightarrow B_{2}^{*}$.

This homomorphism gives a $\log 1$-motif $\left[Y_{2} \rightarrow G_{2, \log }\right]$. Thus, we have an object of $\mathcal{C}_{2}$.

Next, we construct the inverse functor $\delta: \mathcal{C}_{2} \rightarrow \mathcal{C}_{1}^{[\mathrm{pol}]}$.

15.4. Assume that we are given an object of $\mathcal{C}_{2}$. Let $\left[Y_{2} \rightarrow G_{2, \log }\right]$ be the corresponding $\log 1$-motif over $K$ (recall that $K$ has the log structure induced by $M$ ), and let $0 \rightarrow T_{2} \rightarrow G_{2} \rightarrow B_{2} \rightarrow 0$ be the associated exact sequence with $T_{2}$ a torus and $B_{2}$ an abelian variety.

We construct the corresponding object $\left[Y_{1} \rightarrow G_{1, \log }\right]$ of $\mathcal{C}_{1}^{[\mathrm{pol}]}$. By unramified descent, we may and do assume that $Y_{2}$ is constant, that $T_{2}$ splits, and that $B_{2}$ has split semistable reduction.

15.5. Let $\left[Y_{(1)} \rightarrow G_{(1), \log }\right]$ be the object of $\mathcal{C}_{1}^{0,[\mathrm{pol}]}$ corresponding to the object $B_{2}$ of $\mathcal{C}_{2}^{0}$. Let $\left[X_{(1)} \rightarrow G_{(1), \log }^{*}\right]$ be the dual. We have $B_{2}(K)=Y_{(1)} \backslash$ $G_{(1), \log }^{\left(Y_{(1)}\right)}\left(O_{K}\right)$ by [20, Théorème 1] and by Section 12.3 , equation $(*)$. Let 
$0 \rightarrow T_{(1)} \rightarrow G_{(1)} \rightarrow B_{1} \rightarrow 0$ be the exact sequence with $T_{(1)}$ a torus, and let $B_{1}$ be an abelian scheme over $O_{K}$.

15.6. We construct $Y_{1}$. Consider the composition $Y_{2} \rightarrow G_{2, \log } \rightarrow B_{2}$. This induces $Y_{2} \rightarrow B_{2}(K)=Y_{(1)} \backslash G_{(1), \log }^{\left(Y_{(1)}\right)}\left(O_{K}\right)$. Define $Y_{1}$ to be the fiber product of $Y_{2} \rightarrow B_{2}(K) \leftarrow G_{(1), \log }^{\left(Y_{(1)}\right)}\left(O_{K}\right)$.

We obtain a homomorphism $Y_{1} \rightarrow B_{1}$ as the composition $Y_{1} \rightarrow G_{(1), \log }^{\left(Y_{(1)}\right)} \rightarrow$ $B_{1}$.

From the dual $\log 1$-motif $\left[X_{2} \rightarrow G_{2, \log }^{*}\right]$ of $\left[Y_{2} \rightarrow G_{2, \log }\right]$ with an exact sequence $0 \rightarrow T_{2}^{*} \rightarrow G_{2}^{*} \rightarrow B_{2}^{*} \rightarrow 0$ ( $T_{2}^{*}$ is a torus, and $B_{2}^{*}$ is an abelian variety), we obtain similarly $B_{1}^{*}, X_{1}$, and $X_{1} \rightarrow B_{1}^{*}$ in place of $B_{1}, Y_{1}$, and $Y_{1} \rightarrow B_{1}$. This $B_{1}^{*}$ is the dual abelian scheme of $B_{1}$.

Let $T_{1}$ be the torus over $O_{K}$ whose character group is $X_{1}$, and let $T_{1}^{*}$ be the torus over $O_{K}$ whose character group is $Y_{1}$.

15.7. We define $G_{1}$ as the extension of $B_{1}$ by $T_{1}$ corresponding to the homomorphism $X_{1} \rightarrow B_{1}^{*}$. We define $G_{1}^{*}$ as the extension of $B_{1}^{*}$ by $T_{1}^{*}$ corresponding to the homomorphism $Y_{1} \rightarrow B_{1}$. Let $G_{1}^{\prime} \subset G_{1, \log }\left(O_{K}\right)$ be the pullback of $G_{(1), \log }^{\left(Y_{(1)}\right)}\left(O_{K}\right)$ by $G_{1, \log }\left(O_{K}\right) \rightarrow G_{(1), \log }\left(O_{K}\right)$.

Lemma 15.8. We have a Cartesian commutative diagram

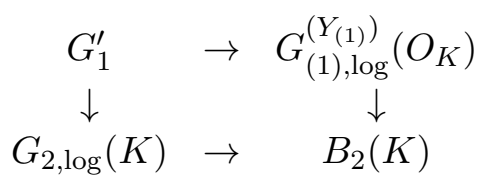

Proof. Step 1. We first give a remark on the map $G_{(1), \log }^{\left(Y_{(1)}\right)}\left(O_{K}\right) \rightarrow B_{2}(K)$. Let $\alpha \in G_{(1), \log }^{\left(Y_{(1)}\right)}\left(O_{K}\right)$, and let $\beta \in B_{2}(K)$ be the image. Let $0 \rightarrow \mathbb{G}_{m} \rightarrow$ $E_{\alpha} \rightarrow B_{1}^{*} \rightarrow 0$ be the extension over $O_{K}$ corresponding to the image of $\alpha$ in $B_{1}\left(O_{K}\right)$, and let $0 \rightarrow \mathbb{G}_{m} \rightarrow E_{\beta} \rightarrow B_{2}^{*} \rightarrow 0$ be the extension over $K$ corresponding to $\beta$. Then $\alpha$ defines a homomorphism $s_{\alpha}: X_{(1)} \rightarrow E_{\alpha, \log }$ which lifts $X_{(1)} \rightarrow B_{1}^{*}$, where $E_{\alpha, \log }$ is the pushout of $\mathbb{G}_{m, \log } \leftarrow \mathbb{G}_{m} \rightarrow E_{\alpha}$. Let $E_{\alpha, \log }^{\prime}$ be the fiber product of $E_{\alpha, \log } \rightarrow B_{1}^{*} \leftarrow\left(G_{(1), \log }^{*}\right)^{\left(X_{(1)}\right)}$. The canonical homomorphism $X_{(1)} \rightarrow\left(G_{(1), \log }^{*}\right)^{\left(X_{(1)}\right)}$ and $s_{\alpha}$ induce a homomorphism $X_{(1)} \rightarrow E_{\alpha, \log }^{\prime}$. Consider the exact sequence

(a) $0 \rightarrow \mathbb{G}_{m, \log } \rightarrow X_{(1)} \backslash E_{\alpha, \log }^{\prime} \rightarrow X_{(1)} \backslash\left(G_{(1), \log }^{*}\right)^{\left(X_{(1)}\right)} \rightarrow 0$. 
On the other hand, let

(b) $0 \rightarrow \mathbb{G}_{m, \log } \rightarrow E_{\beta, \log } \rightarrow B_{2}^{*} \rightarrow 0$ be the exact sequence induced from the extension $E_{\beta}$. We have easily the following.

Claim. There is a canonical isomorphism between the $K$-valued point of the exact sequence ( $a$ ) and the $K$-valued point of the exact sequence (b) which induces the identity map of $\mathbb{G}_{m, \log }(K)$ and the canonical isomorphism $\left(X_{(1)} \backslash\left(G_{(1), \log }^{*}\right)^{\left(X_{(1)}\right)}\right)(K) \cong B_{2}^{*}(K)$.

Step 2. Next we define the map $G_{1}^{\prime} \rightarrow G_{2, \log }(K)$. An element of $G_{1}^{\prime}$ is identified with $(\alpha, s)$, where $\alpha$ is an element of $G_{(1), \log }^{\left(Y_{(1)}\right)}\left(O_{K}\right)$ and $s$ is a homomorphism $X_{1} \rightarrow E_{\alpha, \log }$ which extends $s_{\alpha}: X_{(1)} \rightarrow E_{\alpha, \log }$. It induces a homomorphism $X_{1} \rightarrow E_{\alpha, \log }^{\prime}$, and hence, $X_{2}=X_{1} / X_{(1)} \rightarrow X_{(1)} \backslash E_{\alpha, \log }^{\prime}$. By identifying $\left(X_{(1)} \backslash E_{\alpha, \log }^{\prime}\right)(K)$ with $E_{\beta, \log }(K)$ via the canonical isomorphism of the above claim, we obtain a homomorphism $X_{2} \rightarrow E_{\beta, \log }$ which lifts $X_{2} \rightarrow B_{2}^{*}$. This defines an element of $G_{2, \log }(K)$.

Step 3. We prove that the diagram in Lemma 15.8 is commutative and Cartesian. The commutativity is clear from the definition of the left vertical arrow given in step 2. Since the horizontal arrows are surjective (modulo unramified extensions), the diagram is Cartesian because the kernels of the two horizontal arrows are both identified with $\mathcal{H o m}\left(X_{2}, M^{\text {gp }}\right)(K)$.

15.9. We define a homomorphism $Y_{1} \rightarrow G_{1, \log }$ by Lemma 15.8. This $\left[Y_{1} \rightarrow G_{1, \log }\right]$ is the object of $\mathcal{C}_{1}$ which we want to define. We show that this object is partially polarizable.

Let $p: B_{2} \rightarrow B_{2}^{*}$ be a polarization. Then $p$ induces a morphism $\left[Y_{(1)} \rightarrow\right.$ $\left.G_{(1), \log }\right] \rightarrow\left[X_{(1)} \rightarrow G_{(1), \log }^{*}\right]$. It is easy to prove that the last morphism is a polarization.

Thus, we defined the functor $\delta: \mathcal{C}_{2} \rightarrow \mathcal{C}_{1}^{[\mathrm{pol}]}$. By constructions, it is easily seen that $\delta \gamma \simeq 1$ on $\mathcal{C}_{1}^{[\mathrm{pol}]}$ and $\gamma \delta \simeq 1$ on $\mathcal{C}_{2}$. Hence, we obtain the following.

THEOREM 15.10. We have an equivalence of categories

$$
\mathcal{C}_{1}^{[\mathrm{pol}]} \simeq \mathcal{C}_{2}
$$

given by the functors $\gamma$ and $\delta$ which are the inverses of each other.

\section{§16. Weak log abelian varieties over complete discrete valuation rings, IV}

Here we discuss the rigid analytic theory and related things. 
Our theory of weak log abelian varieties gives the rigid analytic shape of a Tate curve in an algebraic way. Thus, this theory is strikingly near to rigid analytic theory. Hence, it is very important to consider the relation of the theory of weak log abelian variety and rigid analytic theory.

16.1. Let the notation be as in Section 13 .

Let $\mathcal{C}_{3}$ be the category of contravariant functors from the category of $f$ $\log$ rigid analytic spaces over $K$ with the log structure $N$ to the category of commutative groups. Let (WLAV $\mathrm{Wn}^{\mathrm{a}} / K$ ) be the full subcategory of $\mathcal{C}_{3}$ consisting of rigid analytic weak log abelian variety over $K$ with respect to the $\log$ structure $N$. A rigid analytic weak log abelian variety over a general base is defined in a manner similar to the definition of weak log abelian variety in Section 1. But, in what follows, only those over $K$ appear, and since $\operatorname{Spec}(K)$ has a constant log structure, a rigid analytic weak log abelian variety over $K$ corresponds to a rigid analytic log 1-motif (see Section 1.7). Hence, we adopt here the following definition. An object $A$ of $\mathcal{C}_{3}$ is a rigid analytic weak log abelian variety if $A$ can be presented as a quotient of $Y \rightarrow\left(G^{\mathrm{an}}\right)_{\log }^{(Y)}$ for some locally constant sheaf $Y$ of finitely generated and free abelian groups, an (algebraic) semiabelian variety $G$ over $K$, and a homomorphism $Y \rightarrow\left(G^{\text {an }}\right)_{\log }$ such that the induced pairing $X \times Y \rightarrow \mathbb{G}_{m, \log }^{\text {an }} / \mathbb{G}_{m}^{\text {an }}$ $\left(X=\mathcal{H o m}\left(T, \mathbb{G}_{m}\right)^{\text {an }}\right)$ is admissible and nondegenerate.

16.2. We have the following functors $\beta_{1}: \mathcal{C}_{1} \rightarrow \mathcal{C}_{3}$ and $\beta_{2}:(\mathrm{WLAV} / K) \rightarrow$ $\left(\mathrm{WLAV}^{\mathrm{an}} / K\right)$.

Here $\beta_{2}$ is the evident functor. (Note that any object of $(\mathrm{WLAV} / K)$ is with constant degeneration (see Section 1.7).)

The definition of $\beta_{1}$ is as follows. For an object $\left[Y \rightarrow G_{\log }\right]$ of $\mathcal{C}_{1}$, we have the object $A$ of $\mathcal{C}_{3}$ defined to be the sheafification of the functor $U \mapsto$ $Y \backslash\left(G_{K}^{\mathrm{an}}\right)_{\log }^{(Y)}(U)$. It sends $\mathcal{C}_{1}^{[\mathrm{pol}]}$ into $\left(\mathrm{WLAV}^{\mathrm{an}} / K\right)$.

\section{TheOREM 16.3. We have that}

$$
\beta_{2}:(\mathrm{WLAV} / K) \stackrel{\sim}{\rightarrow}\left(\mathrm{WLAV}^{\mathrm{an}} / K\right)
$$

Proof. Since $\left(G^{\mathrm{an}}\right)_{\log }(K)=G_{\log }(K)$ for a semiabelian variety $G$ over $K$, we see that all objects of (WLAV $\left.{ }^{a n} / K\right)$ come from $(\mathrm{WLAV} / K)$.

It remains to prove that the functor $\beta_{2}$ is fully faithful. Let $A, A^{\prime}$ be objects in (WLAV $/ K)$. In the following, we denote by primes the corresponding objects for $A^{\prime}$ to those for $A$. For example, let $\left[Y \rightarrow G_{\log }^{(Y)}\right]$ be the 
$\log 1$-motif associated with $A$. Then, $\left[Y^{\prime} \rightarrow G_{\log }^{\left(Y^{\prime}\right)}\right]$ is the $\log 1$-motif associated with $A^{\prime}$. Let $0 \rightarrow T \rightarrow G \rightarrow B \rightarrow 0$ be the exact sequence with $T$ a torus, and let $B$ be an abelian variety.

Assume that a morphism $h: A^{\text {an }} \rightarrow\left(A^{\prime}\right)^{\text {an }}$ is given, and we prove that it naturally induces a morphism $A \rightarrow A^{\prime}$. First $h$ induces a morphism of $\log 1$ motifs $\left[Y \rightarrow\left(G^{\mathrm{an}}\right)_{\log }^{(Y)}\right]$ to $\left[Y^{\prime} \rightarrow\left(G^{\prime \text { an }}\right)_{\log }^{\left(Y^{\prime}\right)}\right]$ (see [9, Theorem 8.1]). Next, since we can prove that $G^{\text {an }} \rightarrow\left(G^{\prime \text { an }}\right)_{\log }^{\left(Y^{\prime}\right)} / G^{\prime \text { an }}$ is zero (see [9, Corollary 6.1.2]), $h$ induces the morphism $h_{1}: G^{\text {an }} \rightarrow G^{\text {an }}$.

We claim here the following.

Claim. The morphism $\left(T^{\mathrm{an}}\right)_{\mathrm{log}}^{(Y)} \rightarrow B^{\prime a n}$ induced by $h$ is zero.

Proof. We may assume that we are in the situation of Sections 2.4 and 2.5. It is sufficient to prove that the induced homomorphism on the Lie algebras is zero. Let $R=K[[t]]$, let $m$ be its maximal ideal, and let $n$ be a positive integer. By the same argument as in [8, Proposition 3.5.6] or as in [9, Proposition $7.9(3)]$, for any finitely generated subcone $\Delta$ of $C$ (see Section 2.2), there is a bigger finitely generated subcone $\Delta_{1}$ of $C$ such that the inclusion map from the $\Delta$-part $V(\Delta)^{\text {an }}$ of $\left(T^{\mathrm{an}}\right)_{\log }^{(Y)}\left(R / m^{n}\right)$ to the $\Delta_{1}$-part $V\left(\Delta_{1}\right)^{\text {an }}$ of $\left(T^{\text {an }}\right)_{\log }^{(Y)}\left(R / m^{n}\right)$ factors through $\left(T^{\text {an }}\right)_{\log }^{(Y)}(R / m)$. Thus, $\left(T^{\mathrm{an}}\right)_{\log }^{(Y)}\left(R / m^{n}\right) \rightarrow B^{\prime \mathrm{an}}\left(R / m^{n}\right)$ factors through $B^{\prime a n}(R / m)$. Hence, the map on the Lie algebras is zero. Thus, the claim is proved.

By this claim, we see that $h_{1}$ sends $T^{\text {an }}$ into $\left(T^{\prime}\right)^{\text {an }}$ so that we have a homomorphism from an exact sequence $0 \rightarrow T^{\text {an }} \rightarrow G^{\text {an }} \rightarrow B^{\text {an }} \rightarrow 0$ to $0 \rightarrow\left(T^{\prime}\right)^{\text {an }} \rightarrow\left(G^{\prime}\right)^{\text {an }} \rightarrow\left(B^{\prime}\right)^{\text {an }} \rightarrow 0$. It suffices to show that it comes from a homomorphism of exact sequences without the superscripts "an". (Note that $\operatorname{Hom}\left(G, G^{\prime}\right) \rightarrow \operatorname{Hom}\left(G^{\text {an }},\left(G^{\prime}\right)^{\text {an }}\right)$ is not necessarily bijective because there is a nontrivial morphism from a torus to an abelian variety in the category of rigid analytic spaces.)

Consider the exact sequence

$$
\begin{aligned}
0 & \rightarrow \operatorname{Hom}\left(B, T^{\prime}\right) \rightarrow \operatorname{Hom}\left(G, T^{\prime}\right) \rightarrow \operatorname{Hom}\left(T, T^{\prime}\right) \\
& \rightarrow \operatorname{Ext}\left(B, T^{\prime}\right) \rightarrow \operatorname{Ext}\left(G, T^{\prime}\right) \rightarrow \operatorname{Ext}\left(T, T^{\prime}\right) .
\end{aligned}
$$

Consider the homomorphism of exact sequences from this to its "an" version. By using $\operatorname{Hom}\left(B, T^{\prime}\right)=\operatorname{Hom}\left(B^{\text {an }},\left(T^{\prime}\right)^{\text {an }}\right), \operatorname{Hom}\left(T, T^{\prime}\right)=\operatorname{Hom}\left(X^{\prime}, X\right)=$ $\operatorname{Hom}\left(T^{\mathrm{an}},\left(T^{\prime}\right)^{\text {an }}\right), \operatorname{Ext}\left(B, T^{\prime}\right)=\operatorname{Ext}\left(B^{\text {an }},\left(T^{\prime}\right)^{\text {an }}\right)$ (this is because an analytic $\mathbb{G}_{m}$-torsor on $B^{\text {an }}$ is algebraizable), and $\operatorname{Ext}\left(T, T^{\prime}\right)=0$, we have 
(1) $\operatorname{Hom}\left(G, T^{\prime}\right) \rightarrow \operatorname{Hom}\left(G^{\text {an }},\left(T^{\prime}\right)^{\text {an }}\right)$ is surjective, and

(2) $\operatorname{Ext}\left(G, T^{\prime}\right) \rightarrow \operatorname{Ext}\left(G^{\mathrm{an}},\left(T^{\prime}\right)^{\text {an }}\right)$ is injective.

Next, consider the exact sequence

$$
0 \rightarrow \operatorname{Hom}\left(G, T^{\prime}\right) \rightarrow \operatorname{Hom}\left(G, G^{\prime}\right) \rightarrow \operatorname{Hom}\left(G, B^{\prime}\right) \rightarrow \operatorname{Ext}\left(G, T^{\prime}\right)
$$

and a homomorphism of exact sequences from it to its "an" version. Now $h_{1}$ is in $\operatorname{Hom}\left(G^{\text {an }},\left(G^{\prime}\right)^{\text {an }}\right)$, and its image $h_{2}$ in $\operatorname{Hom}\left(G^{\text {an }},\left(B^{\prime}\right)^{\text {an }}\right)$ comes from $\operatorname{Hom}\left(B^{\text {an }},\left(B^{\prime}\right)^{\text {an }}\right)=\operatorname{Hom}\left(B, B^{\prime}\right)$. Hence, $h_{2}$ comes from $\operatorname{Hom}\left(G, B^{\prime}\right)$. By $(1)$ and (2), we see that $h_{1}$ comes from $\operatorname{Hom}\left(G, G^{\prime}\right)$. The proof of Theorem 16.3 is completed.

LEMMA 16.4. We have that $\beta_{1} \simeq \beta_{2} \gamma$ on $\mathcal{C}_{1}^{[\mathrm{pol}]}$.

Proof. Let $C=\left[Y_{1} \rightarrow G_{1, \log }\right]$ be an object of $\mathcal{C}_{1}^{[\mathrm{pol}]}$, and let $\left[Y_{2} \rightarrow G_{2, \log }\right]$ be the $\log 1$-motif over $K$ corresponding to $\gamma(C)$. We have a natural isomorphism

$$
Y_{(1)} \backslash\left(G_{1, K}^{\mathrm{an}}\right)_{\log }^{\left(Y_{1}\right)} \cong\left(G_{2}^{\mathrm{an}}\right)_{\log }^{\left(Y_{2}\right)} .
$$

Hence, we have natural isomorphisms

$$
\beta_{1}(C) \cong Y_{1} \backslash\left(G_{1, K}^{\mathrm{an}}\right)_{\log }^{\left(Y_{1}\right)} \cong Y_{2} \backslash\left(G_{2}^{\mathrm{an}}\right)_{\log }^{\left(Y_{2}\right)} \cong \beta_{2} \gamma(C) .
$$

LEMmA 16.5. We have that $\beta_{2} \simeq \beta_{1} \delta$ on $\mathcal{C}_{2}$.

Proof. By applying $\delta$ to Lemma 16.4 from the right, we have $\beta_{1} \delta \simeq \beta_{2} \gamma \delta \simeq$ $\beta_{2}$ by Theorem 15.10.

Proposition 16.6. The functor $\beta_{1}: \mathcal{C}_{1} \rightarrow \mathcal{C}_{3}$ is fully faithful.

Proof. For an object $\left[Y \rightarrow G_{\log }\right]$ of $\mathcal{C}_{1}$ and $A=\beta_{1}\left(\left[Y \rightarrow G_{\log }\right]\right), Y$ is recovered as the $\mathbb{Z}$-dual of $\mathcal{E x t}^{1}(A, \mathbb{Z})$, and $\left(G_{K}^{\text {an }}\right)_{\log }^{(Y)}$ is obtained as the natural $Y$-torsor of $A$ (see [9, Section 8.2]). The full faithfulness is shown by using these.

REMARK. The above proposition implies that the induced functor $\mathcal{C}_{1}^{[\mathrm{pol}]} \rightarrow$ $\mathcal{C}_{3}$ is fully faithful. The last full faithfulness also follows from Theorem 16.3, $\beta_{2}=\beta_{1} \delta$, and that $\delta$ is an equivalence.

Next we prove the following.

Proposition 16.7. We have that $\beta_{1} \alpha_{1} \simeq \beta_{2} \alpha_{2}$ on $\mathcal{C}_{0}$. 
We prove this proposition in 16.8-16.11. The following lemma is easy, and we omit its proof.

Lemma 16.8. Let $P \rightarrow Q$ be an open immersion of proper rigid analytic spaces. Then, $P$ is an open and closed subspace of $Q$. In particular, if $Q$ is connected, then $P=Q$.

16.9. Let $\mathcal{C}_{0}^{\prime}$ be the category of log algebraic spaces over $O_{K}$ in the second sense (see Section 10.1), let $\mathcal{C}_{1}^{\prime}$ be the category of the formal version of log algebraic spaces in the second sense over $O_{K}$, that is, the inverse limit of the categories of log algebraic spaces in the second sense over $O_{K} / m_{K}^{n}(n \geq 1)$, let $\mathcal{C}_{2}^{\prime}$ be the category of log algebraic spaces over $K$ in the second sense, and let $\mathcal{C}_{3}^{\prime}$ be the category of the rigid analytic version of log algebraic spaces in the second sense over $K$. We have the natural functors $\mathcal{C}_{0}^{\prime} \stackrel{\alpha_{1}}{\rightarrow} \mathcal{C}_{1}^{\prime} \stackrel{\beta_{1}}{\rightarrow} \mathcal{C}_{3}^{\prime}$ and $\mathcal{C}_{0}^{\prime} \stackrel{\alpha_{2}}{\rightarrow} \mathcal{C}_{2}^{\prime} \stackrel{\beta_{2}}{\rightarrow} \mathcal{C}_{3}^{\prime}$. They are compatible with the previous functors $\alpha_{1}, \beta_{1}, \alpha_{2}, \beta_{2}$ and denoted by the same letters.

In this paragraph, we prove that, for a log algebraic space $A \in \mathcal{C}_{0}^{\prime}$ over $O_{K}$ in the second sense, we have a functorial morphism $\beta_{1} \alpha_{1} A \rightarrow \beta_{2} \alpha_{2} A$ in $\mathcal{C}_{3}^{\prime}$ which represents open immersions of rigid analytic spaces.

First, we prove that this is valid for fs $\log$ schemes over $O_{K}$. It reduces to the case of an affine $n$-space $U=\operatorname{Spec} O_{K}\left[T_{1}, \ldots, T_{n}\right]$. Since the concerned functors preserve the product, we may assume that $n=1$. Then, $\beta_{1} \alpha_{1}(U)$ is $O_{K}$ in $K=(U)^{\text {an }}=\beta_{2} \alpha_{2}(A)$, which is an open subspace.

Next, by taking an fs log scheme cover, the functorial morphisms in the case of fs $\log$ schemes induce a functorial morphism for a general $A$ by passing to the quotient. We prove that it represents open immersions.

Let $A \in \mathcal{C}_{0}^{\prime}$ be a log algebraic space over $O_{K}$ in the second sense. Let $T_{2} \rightarrow \beta_{2} \alpha_{2} A$ be a morphism from a rigid analytic space over $K$. Let $T_{1}$ be the fiber product of $T_{2} \rightarrow \beta_{2} \alpha_{2} A \leftarrow \beta_{1} \alpha_{1} A$ as a sheaf. We show that $T_{1} \rightarrow T_{2}$ is represented by an open immersion. Since $A$ is a log algebraic space over $O_{K}$ in the second sense, we can take an fs log scheme $U$ and a surjective morphism $U \rightarrow A$ of étale sheaves, which is log étale. Let $T_{2, U}$ be the base change $T_{2} \times_{\beta_{2} \alpha_{2} A} \beta_{2} \alpha_{2} U$ as a sheaf. Since $T_{2, U} \rightarrow T_{2}$ is a surjection of étale sheaves, we may assume that there is a section $T_{2} \rightarrow T_{2, U}$ of $T_{2, U} \rightarrow T_{2}$. In this case, $T_{2} \rightarrow \beta_{2} \alpha_{2} A$ factors through $\beta_{2} \alpha_{2} U$ so that $T_{2, U}$ is a log algebraic space in the first sense (see Proposition 10.5). Since $T_{1} \times_{\beta_{2} \alpha_{2} A} \beta_{2} \alpha_{2} U \rightarrow T_{2, U}$ is an open immersion, by pulling back the section, we see that $T_{1} \rightarrow T_{2}$ is an open immersion. 
Now, let $A \in \mathcal{C}_{0}$.

Lemma 16.10. Let $\Sigma$ be a complete fan. Then $\beta_{1} \alpha_{1}\left(A^{(\Sigma)}\right)=\beta_{2} \alpha_{2}\left(A^{(\Sigma)}\right)$.

Proof. By Theorem 8.1, $A^{(\Sigma)} \in \mathcal{C}_{0}^{\prime}$, and Section 16.9 implies that $\beta_{1} \alpha_{1}\left(A^{(\Sigma)}\right)$ is an open subspace of $\beta_{2} \alpha_{2}\left(A^{(\Sigma)}\right)$. Furthermore, for $i=1,2$, by Proposition 12.1, $\alpha_{i}\left(A^{(\Sigma)}\right)$ is proper. Hence, $\beta_{i} \alpha_{i}\left(A^{(\Sigma)}\right)$ is proper. On the other hand, $\beta_{2} \alpha_{2}\left(A^{(\Sigma)}\right)$ is connected in the sense of rigid analytic space (see Lemma 12.6). By Lemma 16.8, we have the desired equality.

16.11. In this paragraph, we complete the proof of $\beta_{1} \alpha_{1}(A)=\beta_{2} \alpha_{2}(A)$. It suffices to prove that $\beta_{1} \alpha_{1} A \rightarrow \beta_{2} \alpha_{2} A$ represents surjections of rigid analytic spaces, for we already know that it represents open immersions by Theorem 10.4(1) and Section 16.9. (The surjectivity here is not the surjectivity of a morphism of sheaves.)

Consider any morphism $T_{2} \rightarrow \beta_{2} \alpha_{2} A$ from a rigid analytic space. Let $T_{1}$ be the fiber product of $T_{2} \rightarrow \beta_{2} \alpha_{2} A \leftarrow \beta_{1} \alpha_{1} A$. We have to prove that the induced morphism $T_{1} \rightarrow T_{2}$ of rigid analytic spaces is surjective. Take a complete fan $\Sigma$, let $T_{2}^{\prime}$ be the fiber product of $T_{2} \rightarrow \beta_{2} \alpha_{2} A \leftarrow \beta_{2} \alpha_{2} A^{(\Sigma)}$, and let $T_{1}^{\prime}$ be the fiber product of $T_{2} \rightarrow \beta_{2} \alpha_{2} A \leftarrow \beta_{1} \alpha_{1} A^{(\Sigma)}$. Then $T_{1}^{\prime} \rightarrow T_{2}^{\prime}$ is an isomorphism because $\beta_{1} \alpha_{1}\left(A^{(\Sigma)}\right)=\beta_{2} \alpha_{2}\left(A^{(\Sigma)}\right)$ by Lemma 16.10. Furthermore, $T_{2}^{\prime} \rightarrow T_{2}$ is surjective set-theoretically as a map of rigid analytic spaces. Hence, $T_{1}^{\prime} \rightarrow T_{2}$ is surjective set-theoretically, and hence, $T_{1} \rightarrow T_{2}$ is surjective set-theoretically. This completes the proof of Proposition 16.7.

The following will be used in a forthcoming part of this series of papers.

Proposition 16.12. We have that

(1) $\alpha_{1} \simeq \delta \alpha_{2}$ on $\mathcal{C}_{0}$ and

(2) $\alpha_{2} \simeq \gamma \alpha_{1}$ on $\mathcal{C}_{0}$.

Proof. (1) On $\mathcal{C}_{0}$, we have $\beta_{1} \alpha_{1} \simeq \beta_{2} \alpha_{2} \simeq \beta_{1} \delta \alpha_{2}$, where the first isomorphism follows from Proposition 16.7 and the second isomorphism follows from Lemma 16.5. Then apply Proposition 16.6.

(2) On $\mathcal{C}_{0}$, we have $\beta_{2} \alpha_{2} \simeq \beta_{1} \alpha_{1} \simeq \beta_{2} \gamma \alpha_{1}$, where the first isomorphism follows from Proposition 16.7 and the second isomorphism follows from Lemma 16.4. Then apply Theorem 16.3.

\section{$\S 17$. Proper models}

In this section, we prove the main theorem in this article that a complete fan yields a proper model. This is the algebraic correspondent of the result in [8, Section 5] for analytic log abelian varieties. 
THEOREM 17.1. Let $A$ be a weak log abelian variety over an $f_{s} \log$ scheme S. Assume that we are in the situation of Sections 2.4 and 2.5. Let $\Sigma$ be a complete fan. Then, the algebraic space $A^{(\Sigma)}$ with an $f$ s log structure is proper and log smooth over $S$.

Proof. By Proposition 11.3, it is enough to show that Proposition 11.3(*) is satisfied. Let the notation be as in Proposition 11.3. Then, $A\left(O_{K}\right)=$ $\left(\alpha_{1}(A)\right)\left(O_{K}\right)=\left(\beta_{1} \alpha_{1}(A)\right)(K)=\left(\beta_{2} \alpha_{2}(A)\right)(K)=\left(\alpha_{2}(A)\right)(K)=A(K)$. Here we used $\beta_{1} \alpha_{1}=\beta_{2} \alpha_{2}$ (see Proposition 16.7). Thus Proposition 11.3(*) is satisfied.

Since a complete fan exists, we have the following.

Corollary 17.2. A proper model of A exists.

17.3. A log algebraic space $X$ in the second sense over an fs log scheme $S$ (see Section 10.1) is proper if the following condition is satisfied.

(*) Let $K$ be a complete discrete valuation field. Let $N$ be an fs $\log$ structure on $\eta=\operatorname{Spec}(K)$. Suppose that $N$ is charted by the stalk of $N / \mathcal{O}_{\eta}^{\times}$. Let $M$ be the direct image log structure of $N$ on $\operatorname{Spec}\left(O_{K}\right)$. Then, $X\left(O_{K}\right)=$ $X(K)$ for any morphism $\left(\operatorname{Spec}\left(O_{K}\right), M\right) \rightarrow S$ of log schemes.

A weak log abelian variety is proper by the above proof.

Corollary 17.4. Let $S$ be an $f s$ log scheme. Let $\Sigma$ be a complete fan, and let $P=A^{(\Sigma)}$. Then, $P \rightarrow A$ represents proper morphisms.

Proof. It follows from the properness of $P$ and that of $A$.

\section{$\S 18$. Torsion points of weak log abelian varieties}

In this section, using the representability of a model and its properness, we study the torsion points of a weak $\log$ abelian variety $A$. Although $A$ is not representable, its torsion points surprisingly have the same properties as the classical nondegenerate abelian varieties.

Let $S$ be an fs $\log$ scheme, and let $A$ be a weak log abelian variety over $S$. For $n \geq 1$, we set

$$
A[n]:=\operatorname{Ker}(n: A \rightarrow A) .
$$

Let $g=\operatorname{dim} A$.

Proposition 18.1. Let $n \geq 1$.

(1) The sheaf $A[n]$ is represented by an fs log scheme over $S$ which is finite over $S$. 
(2) Kummer log flat locally on $S, A[n]$ is represented by a usual (classical) finite flat group scheme of rank $n^{2 g}$.

(3) If $n$ is invertible over $S$, Kummer log étale locally on $S$, then $A[n]$ is isomorphic to the constant sheaf $(\mathbb{Z} / n \mathbb{Z})^{2 g}$.

This is proved in 18.2-18.7 as follows.

Lemma 18.2. Let $\langle\rangle:, X \times Y \rightarrow \mathcal{S}$ be an admissible pairing as in Section 1.1. Let $N_{1}, N_{2} \in \operatorname{Hom}(\mathcal{S}, \mathbb{N})$, let $y \in Y$, and assume that $\left(N_{1}+N_{2}\right)(\langle-$, $y\rangle)=0$. Then we have $N_{i}(\langle-, y\rangle)=0$ for $i=1,2$.

Proof. For a homomorphism $N: \mathcal{S} \rightarrow \mathbb{N}$ with $N(\langle X, y\rangle)=\{0\}$, the admissibility of $\langle$,$\rangle implies that y \in Y_{\sigma}$, where $\sigma=N^{-1}(0) \cap \mathcal{S}$. So the lemma easily follows from $\left(N_{1}+N_{2}\right)^{-1}(0) \cap \mathcal{S}=\left(N_{1}^{-1}(0) \cap \mathcal{S}\right) \cap\left(N_{2}^{-1}(0) \cap \mathcal{S}\right)$.

Lemma 18.3. Let the situation be as in Sections 2.1 and 2.2. For a face $\sigma$ of $\operatorname{Hom}(\mathcal{S}, \mathbb{N})$ and for $y \in Y$, let $C(\sigma, y)=\{(N, N(\langle-, y\rangle)) ; N \in \sigma\}$. Then these $C(\sigma, y)$ form a fan.

Proof. It is enough to show that, for faces $\sigma, \tau$ of $\operatorname{Hom}(\mathcal{S}, \mathbb{N})$ and for $y, z \in Y, C(\sigma, y) \cap C(\tau, z)$ is a face of $C(\sigma, y)$. We may assume that $z=$ 0. Let $\left(N_{i}, N_{i}(\langle-, y\rangle)\right) \in C(\sigma, y)$ for $i=1,2$. Assume that $\left(N_{1}+N_{2},\left(N_{1}+\right.\right.$ $\left.\left.N_{2}\right)(\langle-, y\rangle)\right) \in C(\tau, 0)$. Then, $N_{1}+N_{2} \in \tau$, which implies that $N_{i} \in \tau$ for each $i$. Further, by Lemma 18.2, $\left(N_{1}+N_{2}\right)(\langle-, y\rangle)=0$ implies that each $N_{i}(\langle-, y\rangle)=0$. Hence, each $\left(N_{i}, N_{i}(\langle-, y\rangle)\right)$ belongs to $C(\tau, 0)$.

Let $\Sigma$ be the fan consisting of all $C(\sigma, y)$, where $y \in Y$ and where $\sigma$ ranges over all faces of $\operatorname{Hom}(\mathcal{S}, \mathbb{N})$.

LEMMA 18.4. The functor $A[n]$ is represented by an algebraic space with an $f$ s log structure.

Proof. Since $A[n]$ is the fiber product of $A^{\left(n^{-1} \Sigma\right) ~} \stackrel{n}{\rightarrow} A^{(\Sigma)} \stackrel{0}{\leftarrow} S$ and $A^{(\Sigma)}$ is represented by an algebraic space with an fs $\log$ structure by Theorem 8.1, $A[n]$ is represented by an algebraic space with an fs log structure. Here $n^{-1} \Sigma$ is the fan $\left\{\frac{1}{n} \sigma \mid \sigma \in \Sigma\right\}$.

18.5. We prove that $A[n]$ is proper. Let $K$ be a complete discrete valuation field. Let $N$ be an fs $\log$ structure on $\eta=\operatorname{Spec}(K)$. Suppose that $N$ is charted by the stalk of $N / \mathcal{O}_{\eta}^{\times}$. Let $M$ be the direct image log structure of $N$ on $\operatorname{Spec}\left(O_{K}\right)$. Let $\left(\operatorname{Spec}\left(O_{K}\right), M\right) \rightarrow S$ be any morphism of log schemes. Then, $A\left(O_{K}\right)=A(K)$, and hence, $A[n]\left(O_{K}\right)=A[n](K)$; that is, $A[n]$ is proper. Furthermore, $A[n]$ is quasifinite. 
By [16, Théorème A.2], if an algebraic space is proper and quasifinite over a base, then it is a scheme and is finite over the base. Hence, $A[n]$ is an fs $\log$ scheme and is finite over $S$.

Thus (1) is proved.

The proofs of (2) and (3) are roughly as follows. Since, as the properties of a finite object, the kfl (Kummer log flat) local classical flatness in (2), and the két (Kummer log étale) local étaleness in (3) can be checked formally (i.e., can be checked over Artin bases), they are reduced to the case of constant degeneration, and then the rest is also reduced.

Precisely, we argue as follows.

18.6. First, $n: \widetilde{A} \rightarrow \widetilde{A}$ is surjective for kfl topology and even for két topology if $n$ is invertible over the base because it is so for the semiabelian part $G$ and for the quotient $\widetilde{A / G}$ (see Section 18.10). Hence, we may and will assume that $\bar{Y} \subset n \widetilde{A}$.

LEMMA 18.7. If $\bar{Y} \subset n \widetilde{A}$, we have that

(i) $A[n]$ is classical and is finite flat of rank $n^{2 g}$; and

(ii) in the case where $n$ is invertible over the base, $A[n]$ is finite étale and locally isomorphic to $(\mathbb{Z} / n \mathbb{Z})^{2 g}$.

Proof. Since $A[n]$ is represented by a scheme which is finite over the base $S$ (see Proposition 18.1(1)), the situation is reduced to the case for each $\operatorname{Spec}\left(\mathcal{O}_{S, s} / m_{s}^{n}\right)$, where $s \in S, m_{s}$ is the maximal ideal of $\mathcal{O}_{S, s}$, and $n \geq 1$, and hence is reduced to the case of constant degeneration. In this case, by the assumption that $\bar{Y} \subset n \widetilde{A}$, we have an exact sequence $0 \rightarrow \widetilde{A}[n] \rightarrow A[n] \rightarrow$ $\bar{Y} / n \bar{Y} \rightarrow 0$. Since $\widetilde{A}[n]=G[n]$, this implies the results.

Thus, Proposition 18.1 is proved.

REMARK 18.8. Proposition 18.1(1), (2) imply that, for any prime number $p$, the subgroup sheaf $\bigcup_{n \geq 0} A\left[p^{n}\right]$ is a log $p$-divisible group over $S$ (see [7], [13]). However, we do not discuss this subject here.

18.9. Finally, we briefly discuss the Tate modules.

Let $s \in S$, and let $l$ be a prime number which is invertible at $s$. Then, the $\log$ fundamental group $\pi_{1}^{\log }(s)$ acts on the $l$-adic Tate module

$$
T_{l}(A)_{\bar{s}(\text { két })}:={\underset{\leftarrow}{n}}_{\lim } A\left[l^{n}\right]_{\bar{s}(\text { két })}
$$

where $\bar{s}$ (két) denotes a log geometric point of the két site over $s$. 
Let $\langle\rangle:, \bar{X}_{\bar{s}} \times \bar{Y}_{\bar{s}} \rightarrow\left(M_{S}^{\mathrm{gp}} / \mathcal{O}_{S}^{\times}\right)_{\bar{s}}$ be the induced admissible pairing at $s$. Then, we have the following two homomorphisms.

18.9.1. The canonical surjective homomorphism $T_{l}(A)_{\bar{s} \text { (két) }} \rightarrow \bar{Y}_{\bar{s}} \otimes \mathbb{Z}_{l}$ coming from the exact sequence $0 \rightarrow \bar{Y}_{\bar{s}} \rightarrow\left(G_{\log }^{(Y)}\right)_{\bar{s}(\text { két })} \rightarrow A_{\bar{s}(\text { két) }} \rightarrow 0$ (we prove the surjectivity below in Section 18.10).

18.9.2. The canonical injective homomorphism $\operatorname{Hom}\left(\bar{X}_{\bar{s}}, \mathbb{Z}_{l}(1)\right) \rightarrow$ $T_{l}(A)_{\bar{s}(\text { két) }}$ coming from the injection $T_{\bar{s}} \rightarrow A_{\bar{s}(\text { két) }}$, which preserve the actions of $\pi_{1}^{\log }(s)$, such that the image of homomorphism 18.9.2 is contained in the kernel of homomorphism 18.9.1.

18.10. We prove the surjectivity of homomorphism 18.9.1, which comes from the $l$-divisibility of $\left(G_{\log }^{(Y)}\right)_{\bar{s}(\text { két) }}$. Since $\left(G_{\log }\right)_{\bar{s} \text { (két) }}$ is $l$-divisible, it is enough to show that, for any $\varphi \in \mathcal{H o m}\left(\bar{X}_{\bar{s}}, \mathbb{G}_{m, \log } / \mathbb{G}_{m}\right)$, if $\varphi^{l}$ belongs to the $(Y)$-part, then $\varphi$ belongs to the $(Y)$-part. It reduces to that, for any $x \in \bar{X}_{\bar{s}}, y \in \bar{Y}_{\bar{s}}$, there exist $y_{1}, y_{2} \in \bar{Y}_{\bar{s}}$ such that $\left\langle x, y_{1}^{l}\right\rangle|\langle x, y\rangle|\left\langle x, y_{2}^{l}\right\rangle$. It further reduces to that the inverse image of $\left(M_{S} / \mathcal{O}_{S}^{\times}\right)_{\bar{s}}$ by $\langle x,-\rangle$ in $\bar{Y}_{\bar{s}}$ generates $\bar{Y}_{\bar{s}}$, which is deduced from the following lemma.

Lemma 18.10.1. Let $\langle\rangle:, X \times Y \rightarrow \mathcal{S}^{\mathrm{gp}}$ be an admissible pairing as in Section 1.1. Let $x \in X$, and let $W=\langle x, Y\rangle$. Then $(W \cap \mathcal{S})^{\mathrm{gp}}=W$.

Proof. Let $\sigma$ be the face of $\mathcal{S}$ spanned by $W_{\mathbb{Q}} \cap \mathcal{S}$.

We prove that there is an $N \in \operatorname{Hom}\left(\mathcal{S}, \mathbb{R}_{\geq 0}\right)$ with kernel $\sigma$ such that $N(W)=0$. First, if $\mathcal{S}$ is sharp and $\sigma=\{1\}$, it is easy to see that we can take such an $N$. The general case is reduced to this case by dividing by $\sigma_{\mathbb{R}}$ if we show that $\mathcal{S} \cap\left(W \sigma^{\mathrm{gp}}\right) \subset \sigma$. Let $s \in \mathcal{S} \cap\left(W \sigma^{\mathrm{gp}}\right)$. We show that $s \in \sigma$. We may assume that $s \in W \sigma$. Hence, there are $w \in W$ and $t \in \sigma$ such that $s=w t$. Since $t$ divides some element of $W \cap \mathcal{S}$ in $\mathcal{S}$, we may assume that $t \in W \cap \mathcal{S}$. In this case, $s \in W \cap \mathcal{S} \subset \sigma$. Thus, we conclude that the desired $N$ exists.

By the admissibility, the induced pairing $X / X_{\sigma} \times Y / Y_{\sigma} \rightarrow \mathbb{R}$ by $N$ is nondegenerate. Hence, $x \in X_{\sigma}$ and $W \subset \sigma^{\mathrm{gp}}$. Since $W$ contains an interior point of $\sigma$, we have $W=(W \cap \sigma)^{\mathrm{gp}}=(W \cap \mathcal{S})^{\mathrm{gp}}$.

We have the following.

Proposition 18.11. Let $\gamma$ be an element of the geometric part $\pi_{1}^{\log }(s)_{\text {geom }}:=\pi_{1}^{\log }(\bar{s})$ of $\pi_{1}^{\log }(s)$. Then $\gamma$ acts trivially on the kernel of homomorphism 18.9.1 and trivially on the cokernel of homomorphism 18.9.2 (so 
the action of $\gamma$ on $T_{l}(A)_{\bar{s}(\mathrm{két})}$ is unipotent), and the homomorphism

$$
\bar{Y}_{\bar{s}} \otimes \mathbb{Z}_{l} \rightarrow \operatorname{Hom}\left(\bar{X}_{\bar{s}}, \mathbb{Z}_{l}(1)\right)
$$

induced by $\gamma-1$ coincides with the map induced by

$$
\bar{X}_{\bar{s}} \otimes \bar{Y}_{\bar{s}} \stackrel{\langle,\rangle}{\rightarrow}\left(M_{S}^{\mathrm{gp}} / \mathcal{O}_{S}^{\times}\right)_{\bar{s}} \stackrel{h}{\rightarrow} \mathbb{Z}_{l}(1) .
$$

Here $h$ is the image of $\gamma$ under the canonical isomorphism

$$
\text { l-part of } \pi_{1}^{\log }(s)_{\text {geom }} \cong \operatorname{Hom}\left(\left(M_{S}^{\mathrm{gp}} / \mathcal{O}_{S}^{\times}\right)_{\bar{s}}, \mathbb{Z}_{l}(1)\right) \text {. }
$$

Proof. We may assume that $S=\bar{s}$. Hereafter in this proof, we identify a sheaf on $S$ with its két stalk with Galois action. Let $n \geq 0$.

Since $T\left[l^{n}\right] \stackrel{\cong}{\rightarrow} T_{\log }\left[l^{n}\right]$, we have $G\left[l^{n}\right] \stackrel{\cong}{\rightarrow} G_{\log }\left[l^{n}\right]$, so that $G\left[l^{n}\right]=G_{\log }^{(Y)}\left[l^{n}\right]$. Thus, the action of $\gamma$ on the kernel of homomorphism 18.9.1 is trivial.

Next, take an element $a$ of $A\left[l^{n}\right]$. We lift it to an element $\widetilde{a}$ of $G_{\log }$. Then, $\widetilde{a}^{l}$ belongs to the image of $Y \rightarrow G_{\log }$. Since $\frac{\gamma(\widetilde{a})}{\widetilde{a}}$ maps to 1 in $B$, it lies in $\operatorname{Hom}\left(X, \mathbb{Z} / l^{n} \mathbb{Z}(1)\right) \subset T$. Thus, the action on the cokernel of homomorphism 18.9.2 is also trivial.

Finally, let $x \in X$, and let $y \in Y$. Let $g$ be the image of $y$ in $G_{\log }$. Then, as above, $\varphi(g):=\frac{\gamma\left(g^{\frac{1}{l^{n}}}\right)}{g^{\frac{1}{l^{n}}}} \operatorname{lies}$ in $\operatorname{Hom}\left(X, \mathbb{Z} / l^{n} \mathbb{Z}(1)\right)$, and we can consider the value $\varphi(g)(x) \in \mathbb{Z} / l^{n} \mathbb{Z}(1)$. Now we claim that this $\varphi(g)(x)$ coincides with $h(\langle x, y\rangle) \bmod l^{n}$. In fact, consider a lift $[]:, X \times Y \rightarrow \mathbb{G}_{m, \log }$ of the pairing $\langle\rangle:, X \times Y \rightarrow \mathbb{G}_{m, \log } / \mathbb{G}_{m}$. Let $t=[-, y] \in T_{\log }$. Then we have $t \bmod T=$ $g \bmod G$ in $\operatorname{Hom}\left(X, \mathbb{G}_{m, \log } / \mathbb{G}_{m}\right)$. Hence, $\varphi(g)=\frac{\gamma\left(t^{1 /\left(l^{n}\right)}\right)}{t^{1 /\left(l^{n}\right)}}$. Substituting $x$, we have $\varphi(g)(x)=\frac{\gamma\left([x, y]^{1 /\left(l^{n}\right)}\right)}{[x, y]^{1 /\left(l^{n}\right)}}$. Since $[x, y] \bmod \mathbb{G}_{m}$ is $\langle x, y\rangle$, the right-hand side of the last equation is equal to $h(\langle x, y\rangle) \bmod l^{n}$. Thus, we conclude that $\varphi(g)(x)=h(\langle x, y\rangle) \bmod l^{n}$, and the last statement of Proposition 18.11 follows.

Acknowledgments. The authors are very grateful to the referees for their careful reading and a list of many useful suggestions.

\section{REFERENCES}

[1] M. Artin, "Algebraization of formal moduli, I" in Global Analysis (Papers in Honor of K. Kodaira), University of Tokyo Press, Tokyo, 1969, 21-71. MR 0260746.

[2] - "The implicit function theorem in algebraic geometry" in Algebraic Geometry (Bombay, 1968), Oxford University Press, London, 1969, 13-34. MR 0262237. 
[3] A. J. de Jong et al., "Morphisms of algebraic spaces" in The Stacks Project, Chapter 51, May 29, 2015, http://stacks.math.columbia.edu/download /spaces-morphisms.pdf.

[4] G. Faltings and C. Chai, Degeneration of Abelian Varieties, Ergeb. Math. Grenzgeb. (3) 22, Springer, Berlin, 1990. MR 1083353. DOI 10.1007/978-3-662-02632-8.

[5] A. Grothendieck, with M. Artin and J.-L. Verdier, Théorie des topos et cohomologie étale des schémas (SGA4), Lecture Notes in Math. 269, Springer, Berlin, 1972. MR 0354652.

[6] A. Grothendieck, Étude globale élémentaire de quelques classes de morphismes (EGA II), Publ. Math. Inst. Hautes Études Sci. 8 (1961). MR 0163909.

[7] L. Illusie, "Logarithmic spaces (according to K. Kato)" in Barsotti Symposium in Algebraic Geometry (Abano Terme, 1991), Perspect. Math. 15, Academic Press, San Diego, Calif., 1994, 183-203. MR 1307397.

[8] T. Kajiwara, K. Kato, and C. Nakayama, Logarithmic abelian varieties, I: Complex analytic theory, J. Math. Sci. Univ. Tokyo 15 (2008), 69-193. MR 2422590.

[9] - Logarithmic abelian varieties, II: Algebraic theory, Nagoya Math. J. 189 (2008), 63-138. MR 2396584.

[10] - Logarithmic abelian varieties, III: Logarithmic elliptic curves and modular curves, Nagoya Math. J. 210 (2013), 59-81. MR 3079275. DOI 10.1215/00277630-2077017.

[11] K. Kato, Toric singularities, Amer. J. Math. 116 (1994), 1073-1099. MR 1296725. DOI $10.2307 / 2374941$.

[12] — Logarithmic structures of Fontaine-Illusie, II: Logarithmic flat topology, preprint, 1991.

[13] - Logarithmic Dieudonné theory, preprint, 1992.

[14] K. Kato and S. Usui, Classifying Spaces of Degenerating Polarized Hodge Structures, Ann. of Math. Stud. 169, Princeton University Press, Princeton, 2009. MR 2465224.

[15] D. Knutson, Algebraic Spaces, Lecture Notes in Math. 203, Springer, Berlin, 1971. MR 0302647.

[16] G. Laumon and L. Moret-Bailly, Champs algébriques, Ergeb. Math, Grenzgeb. (3) 39, Springer, Berlin, 2000. MR 1771927.

[17] C. Nakayama, Logarithmic étale cohomology, Math. Ann. 308 (1997), 365-404. MR 1457738. DOI 10.1007/s002080050081.

[18] W. Niziol, K-theory of log-schemes, I, Doc. Math. 13 (2008), 505-551. MR 2452875.

[19] M. C. Olsson, Log algebraic stacks and moduli of log schemes, Ph.D. dissertation, University of California, Berkeley, Berkeley, Calif., 2001. MR 2702292.

[20] M. Raynaud, "Variétés abéliennes et géométrie rigide" in Actes du Congrès International des Mathématiciens, I (Nice, 1970), Gauthier-Villars, Paris, 1971, 473-477. MR 0427326. 
Takeshi Kajiwara

Department of Applied Mathematics

Faculty of Engineering

Yokohama National University

Hodogaya-ku

Yokohama 240-8501

Japan

kajiwara@ynu.ac.jp

Kazuya Kato

Department of Mathematics

University of Chicago

5734 S. University Avenue

Chicago, Illinois 60637

USA

kkato@math.uchicago .edu

Chikara Nakayama

Department of Economics

Hitotsubashi University

2-1 Naka, Kunitachi

Tokyo 186-8601

Japan

c.nakayama@r.hit-u.ac.jp 